\title{
Oriented Ice Crystals: A Single-Scattering Property Database for Applications to Lidar and Optical Phenomenon Simulations
}

\author{
MASANORI SAITO AND PING YANG \\ Department of Atmospheric Sciences, Texas A\&M University, College Station, Texas
}

(Manuscript received 8 February 2019, in final form 27 May 2019)

\begin{abstract}
A database (TAMUoic2019) of the scattering, absorption, and polarization properties of horizontally oriented hexagonal plates (HOPs) and horizontally oriented hexagonal columns (HOCs) at three wavelengths $(355,532$, and $1064 \mathrm{~nm})$ is developed for applications to radiative transfer simulations and remote sensing implementations involving oriented ice crystals. The maximum dimension of oriented ice crystals ranges from 50 to $10000 \mu \mathrm{m}$ in 165 discrete size bins. The database accounts for 94 incident directions. The single-scattering properties of oriented ice crystals are computed with the physical-geometric optics method (PGOM), which is consistent with the invariant-imbedding T-matrix method for particles with size parameters larger than approximately 100-150. Note that the accuracy of PGOM increases as the size parameter increases. PGOM computes the two-dimensional phase matrix as a function of scattering polar and azimuth angles, and the phase matrix significantly varies with the incident direction. To derive the bulk optical properties of ice clouds for practical radiative transfer applications, the optical properties of individual HOPs and HOCs are averaged over the probability distribution of the tilting angle of oriented ice crystals based on the use of the TAMUoic2019 database. Simulations of lidar signals associated with ice clouds based on the bulk optical properties indicate the importance of the fraction of oriented ice crystals and the probability distribution of the tilting angle. Simulations of optical phenomena caused by oriented ice crystals demonstrate that the computed single-scattering properties of oriented ice crystals are physically rational.
\end{abstract}

\section{Introduction}

Ice clouds are ubiquitous and have significant impacts on the Earth radiation budget and hydrological cycle, which have been extensively studied for several decades based on numerical radiative transfer models (e.g., Liou 1986; Lohmann and Roeckner 1995; Waliser et al. 2009; Yang et al. 2015) and remote sensing techniques (e.g., Platnick et al. 2003; Sassen and Comstock 2001; Sassen et al. 2008; Yang et al. 2018). The singlescattering properties of ice crystals are fundamental to the development of many applications involving ice clouds. The bulk optical properties of ice clouds are the counterparts of an ensemble of ice crystals with certain size and shape distributions (e.g., Baum et al. 2005). The bulk ice cloud optical properties are used for radiative flux simulations involving ice clouds in general circulation models (GCMs) and remote sensing of ice clouds from satellite measurements. At present,

\footnotetext{
Corresponding author: Masanori Saito, masa.saito@tamu.edu
}

several single-scattering property databases of randomly oriented ice crystals are publicly available (Liu 2008; Yang et al. 2013, hereafter Y13; Kuo et al. 2016; Bi and Yang 2017; Ding et al. 2017), which have been widely used for ice cloud retrievals from satellite measurements.

Under low-turbulence conditions, ice crystals with certain particle sizes can be preferentially oriented (particularly horizontally oriented). With certain solar geometric configurations, ice clouds containing oriented ice crystals cause particular optical phenomena such as parhelia (sundogs), circumhorizontal and circumzenithal arcs due to horizontally oriented hexagonal plates (HOPs), and tangent arcs due to horizontally oriented hexagonal columns (HOCs; Greenler 1980; Liou and Yang 2016). This is because the single-scattering properties of specifically oriented ice crystals are substantially different from those of randomly oriented ice crystals that cause $22^{\circ}$ and $46^{\circ}$ halos. Therefore, the presence of oriented ice crystals in clouds has nonnegligible impacts on solar 
reflectances observed from passive satellite sensors (Chepfer et al. 1999; Marshak et al. 2017) and substantial impacts on backscattering signals from lidar measurements (Zhou et al. 2012; Borovoi et al. 2012). To incorporate oriented ice crystals into radiative transfer simulations or ice cloud property retrievals, a singlescattering property database for oriented ice crystals is essential.

Since the size of ice crystals is much larger than the wavelength of visible light, numerically exact and semianalytical methods [e.g., the discrete dipole approximation (DDA; Purcell and Pennypacker 1973), finite-difference time domain method (FDTD; Yee 1966), and T-matrix method (Waterman 1965; Mishchenko and Travis 1994)] for light-scattering calculations are not practically feasible because of the limitation on the applicable size parameter (defined as $\pi D / \lambda$, where $\lambda$ and $D$ are the wavelength and the maximum characteristic dimension of the particle, respectively) range for these methods and their tremendous demand on computational resources. The conventional geometric-optics method (CGOM) calculates the optical properties of ice crystals by directly computing the scattered field in far-field zone (Cai and Liou 1982) based on the principles of geometric optics and Fraunhofer diffraction. In the case of oriented ice particles, CGOM leads to angular singularities of scattered light, which are stemmed from the inherent flaws of the ray-tracing technique. In addition, CGOM is not accurate, particularly for moderate particles, because of the assumption that the extinction efficiency is 2 regardless of the size parameter. Yang and Liou $(1996,1997)$ developed a physical-geometric optics method (PGOM) through mapping the near field to the far field with either an electromagnetic surface integral equation or an electromagnetic volume integral equation. The minimum applicable size parameter of PGOM is smaller than the CGOM counterpart. The computational efficiency of PGOM has been further improved by implementing a broad-beam tracing technique in conjunction with the use of some computer graphics techniques (Bi et al. 2011; Borovoi et al. 2014; Sun et al. 2017). Yang et al. (2019) show that PGOM is consistent with the numerically exact invariant-imbedding T-matrix method (IITM; Johnson 1988; Bi et al. 2013; Bi and Yang 2014) for size parameters 100-150. Furthermore, PGOM is applicable to the computation of the phase matrix in the exact $180^{\circ}$ backscattering direction, whereas the CGOM backscatter is provided as the relevant average in a small angular region near scattering angle $180^{\circ}$.
In this study, we use PGOM to develop a database of the single-scattering properties of HOPs and HOCs (TAMUoic2019) for applications to lidar and radiative transfer simulations involving oriented ice crystals. Section 2 describes the detailed methodology and structure of the present single-scattering property database. Section 3 presents the PGOM-based singlescattering properties of HOPs and HOCs in comparison with the IITM solutions. Section 4 presents the bulk optical properties of ice clouds containing HOPs and HOCs, and applications to lidar and optical phenomena simulations based on the bulk optical properties. Section 5 summarizes this study.

\section{Methods}

\section{a. Ice particle-scattering geometries}

Figure 1 illustrates the Cartesian coordinate systems that determine the scattering and particle geometries. The laboratory coordinate system, $O X_{1} Y_{1} Z_{1}$, has the $X_{1}$ $Y_{1}$ plane being parallel to the surface and the $Z_{1}$ axis pointing to the zenith. The direction of the incident beam is specified in terms of the incident polar angle $\theta_{\text {inc }}$ and azimuth angle $\phi_{\text {inc }}$ (to be $0^{\circ}$ ) based on the laboratory coordinate system (Fig. 1a). The particle coordinate system, $O X_{p} Y_{p} Z_{p}$, defines the particle shape as shown in Fig. 1b. The $Z_{p}$ axis is perpendicular to the basal face of a hexagonal plate/column. The origin $O$ is placed at the center of the particle, and the $X_{p} Y_{p}$ plane is parallel to the basal face and intersects at the middle of the side faces of the particle. The $Y_{p}$ axis intersects two opposite vertices of a hexagon. The shape of a hexagonal plate/column is determined by the half width $a$ of the hexagonal cross section and maximum column length $L$. The scattering coordinate system, $O X_{s} Y_{s} Z_{s}$, defines the direction of the scattered beam in terms of the scattering polar angle $\theta_{\text {sca }}$ and azimuth angle $\phi_{\text {sca }}$ (Fig. 1c). The $Z_{s}$ axis points along the direction of the incident beam. The $X_{s}$ axis is defined on the $Z_{1} Z_{s}$ plane, which satisfies $\mathbf{X}_{s} \cdot \mathbf{Z}_{l} \geq 0$ (Fig. 1a).

To define the orientation of a hexagonal plate or column, the particle coordinate system is rotated from the laboratory coordinate system with the Euler angles $(\alpha, \beta, \gamma)$ based on $Z Y Z$ rotations as shown in Fig. 1d. First, the $O X_{1} Y_{1} Z_{1}$ coordinate system is rotated by $-\alpha$ with respect to the $Z_{1}$ axis to be the $O X^{\prime} Y^{\prime} Z^{\prime}$ coordinate system. Next, the $O X^{\prime} Y^{\prime} Z^{\prime}$ coordinate system is rotated by $-\beta$ with respect to the $Y^{\prime}$ axis to be the $O X^{\prime \prime} Y^{\prime \prime} Z^{\prime \prime}$ coordinate system. Finally, the $O X^{\prime \prime} Y^{\prime \prime} Z^{\prime \prime}$ coordinate system is rotated by $-\gamma$ with respect to the $Z^{\prime \prime}$ axis to form $O X_{p} Y_{p} Z_{p}$ (Fig. 1d). Therefore, the transformation between $O X_{1} Y_{1} Z_{1}$ and $O X_{p} Y_{p} Z_{p}$ is 

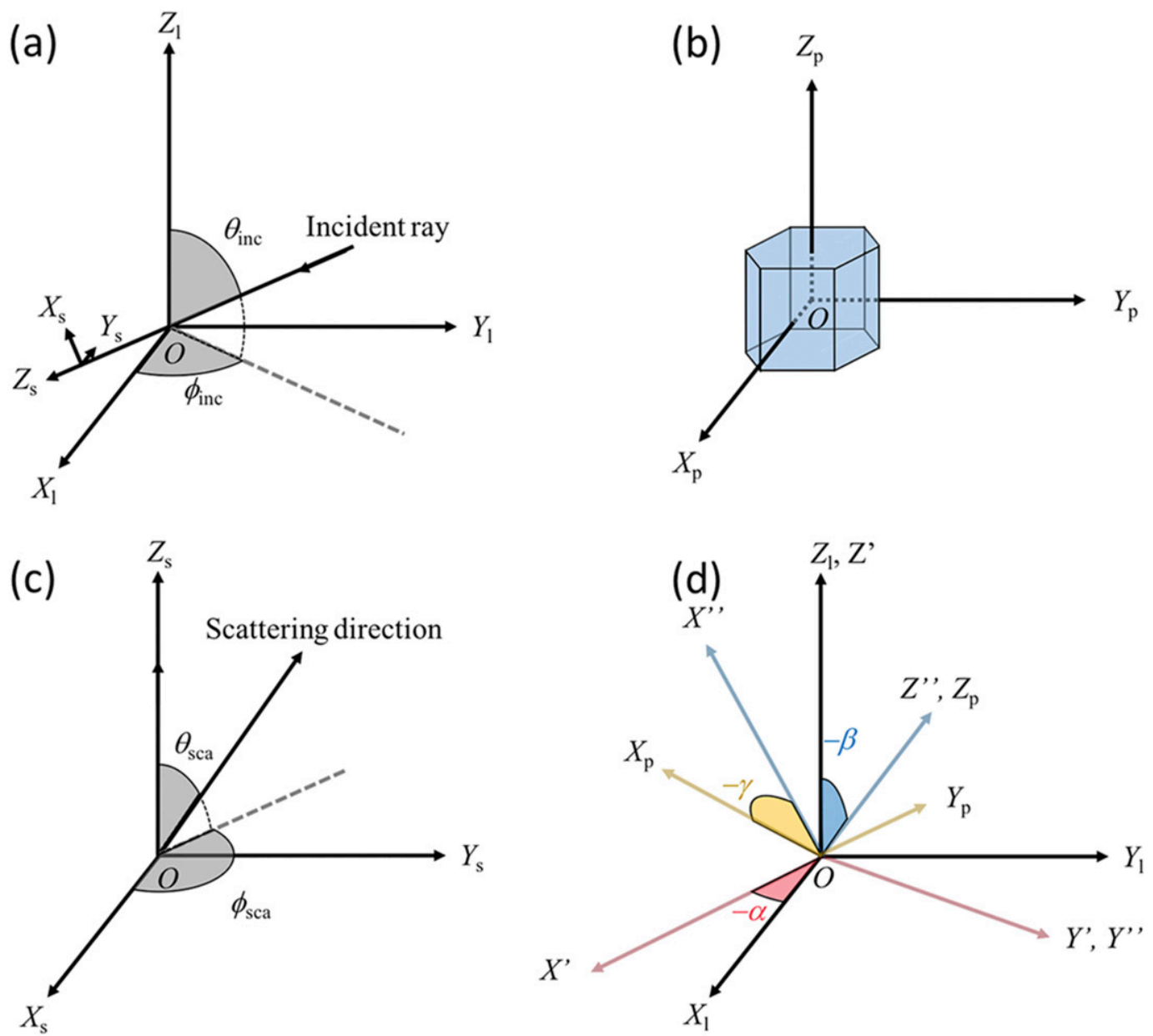

FIG. 1. Illustrations of (a) the laboratory coordinate system (subscript $l$ ), (b) the particle coordinate system (subscript $p$ ), (c) the scattering coordinate system (subscript $s$ ), and (d) a rotation from the laboratory coordinate system to particle coordinate system.

$$
\left(\begin{array}{c}
x_{p} \\
y_{p} \\
z_{p}
\end{array}\right)=\mathbf{R}_{z}(-\gamma) \mathbf{R}_{y}(-\beta) \mathbf{R}_{z}(-\alpha)\left(\begin{array}{c}
x_{l} \\
y_{l} \\
z_{l}
\end{array}\right),
$$

where the rotation matrices $\mathbf{R}_{z}(\Psi)$ around the $z$ axis and $\mathbf{R}_{y}(\Psi)$ around the $y$ axis are given by

$$
\begin{aligned}
& \mathbf{R}_{z}(\Psi)=\left[\begin{array}{ccc}
\cos \Psi & -\sin \Psi & 0 \\
\sin \Psi & \cos \Psi & 0 \\
0 & 0 & 1
\end{array}\right], \\
& \mathbf{R}_{y}(\Psi)=\left[\begin{array}{ccc}
\cos \Psi & 0 & \sin \Psi \\
0 & 1 & 0 \\
-\sin \Psi & 0 & \cos \Psi
\end{array}\right] .
\end{aligned}
$$

To summarize these coordinate systems, the particle shape is defined in reference to the particle coordinate system. The direction of the incident light and the particle orientation are defined in reference to the laboratory coordinate system. The scattering geometry is defined in reference to the scattering coordinate system.

Hexagonal columns and plates have different preferred orientations. In the case of a HOP, the basal face of a hexagonal plate tends to be parallel to the $X_{1} Y_{1}$ plane, but the particle randomly rotates around its symmetric axis. In the case of a HOC, the basal face of a hexagonal column tends to be perpendicular to the $X_{1} Y_{1}$ plane. The long axis of the hexagonal column randomly rotates on the $X_{1} Y_{1}$ plane and rotates around particle symmetry axis. Therefore, in this study the Euler angles are selected to be $\alpha=0^{\circ}, \beta=0^{\circ}$, and $\gamma \in\left[0^{\circ}, 60^{\circ}\right]$ for HOPs, and $\alpha \in\left[0^{\circ}, 180^{\circ}\right], \beta=90^{\circ}$, and $\gamma \in\left[0^{\circ}, 60^{\circ}\right]$ for HOCs. The aspect ratio $(2 a / L)$ is defined, similarly to Y13, as 


$$
2 a / L=\left\{\begin{array}{cl}
1, & a \leq 2 \mu \mathrm{m} \\
0.2914 a+0.4172, & 2<a<5 \mu \mathrm{m} \\
0.8038 a^{0.526}, & a \geq 5(\mu \mathrm{m})
\end{array} \text { for } \quad\right. \text { HOP }
$$

$$
2 a / L=\left\{\begin{array}{cc}
0.7, & L<100 \mu \mathrm{m} \\
6.96 / \sqrt{L}, & L \geq 100 \mu \mathrm{m}
\end{array} \text { for } \mathrm{HOC} .\right.
$$

\section{b. Scattering property calculations}

PGOM computes the single-scattering properties of HOPs and HOCs (Sun et al. 2017; Yang et al. 2019) for each ice crystal orientation. The values of the refractive index of ice used in the present study are taken from Warren and Brandt (2008). The single-scattering properties of oriented ice crystals are obtained by averaging a large number of individual oriented particles rotated with respect to the Euler angles $\alpha$ and $\gamma$ for HOCs and $\gamma$ for HOPs. To enhance the accuracy of the singlescattering properties while reducing the computational burden, the number of particle orientations with respect to the Euler angles varies from 100 to 1680 , depending on the maximum dimension and particle habit.

\section{c. General structure of the database, TAMUoic2019}

Table 1 lists the parameters for the structure of the database. The particle habits in the database include HOP and HOC. The database is developed at three wavelengths (355, 532 , and $1064 \mathrm{~nm}$ ) commonly used for lidar measurements. The particles sizes in the database ranges $50-10000 \mu \mathrm{m}$ in terms of the maximum dimension, which are discretized into 165 size bins. The database includes 94 incident polar angles ranging from $0^{\circ}$ to $90^{\circ}$ with nonuniform intervals, which are $0.01^{\circ}, 0.1^{\circ}, 0.5^{\circ}, 2^{\circ}$, and $5^{\circ}$ for incident polar angles $0-0.3^{\circ}, 0.3^{\circ}-3^{\circ}, 3^{\circ}-10^{\circ}, 10^{\circ}-30^{\circ}$, and $30^{\circ}-90^{\circ}$, respectively. The scattering polar and azimuth angles are discretized into 498 and 180 angular directions. The database contains 1 ) the geometric projected area, 2) particle volume, 3) randomly oriented projected area, 4) extinction efficiency, 5) singlescattering albedo, 6) asymmetry parameter, and 7) phase matrix for each particle habit, wavelength, maximum dimension, and incident polar angle. Each phase matrix in the database has the full 16 elements (i.e., $P_{11}, P_{12}, P_{13}, P_{14}, P_{21}$, $P_{22}, P_{23}, P_{24}, P_{31}, P_{32}, P_{33}, P_{34}, P_{41}, P_{42}, P_{43}$, and $\left.P_{44}\right)$ because the nondiagonal elements of the phase matrix of specifically oriented particle are not zero (Noel et al. 2001).

\section{Results}

\section{a. Comparisons}

Figure 2 shows the two-dimensional phase matrix elements $\left(P_{11}, P_{12} / P_{11}\right.$, and $\left.P_{22} / P_{11}\right)$ of HOPs computed with PGOM and IITM at wavelength $532 \mathrm{~nm}$. The size parameter $(\pi D / \lambda)$ is 120 , corresponding to $D=20.32 \mu \mathrm{m}$. The incident polar angle is $60^{\circ}$ in this case. The overall angular patterns of the computed two-dimensional phase matrix elements are consistent between PGOM and IITM except for stronger geometric optics features of $P_{11}$ computed with PGOM than the IITM counterparts. Bi et al. (2011) compare the two-dimensional phase matrix elements of HOPs between the previous version of PGOM and DDA (Yurkin et al. 2007). It shows a reasonable consistency for absorbing HOPs but some deviations between PGOM and DDA calculations for nonabsorbing HOPs with size parameter 50 (corresponding to $D=$ $8.47 \mu \mathrm{m}$ at $532 \mathrm{~nm}$ ). Figure 3 shows the differences of the phase matrix elements of a HOP between IITM and PGOM. These differences $\chi_{i j}$ are defined as follows:

$$
\chi_{i j}=\frac{\int_{0}^{2 \pi} \int_{0}^{\pi} \Delta P_{i j}^{2} \sin \theta_{\mathrm{sca}} d \theta_{\mathrm{sca}} d \phi_{\mathrm{sca}}}{\int_{0}^{2 \pi} \int_{0}^{\pi} \sin \theta_{\mathrm{sca}} d \theta_{\mathrm{sca}} d \phi_{\mathrm{sca}}},
$$

where $\Delta P_{i j}$ is $\ln P_{11, \mathrm{PGOM}}\left(\theta_{\mathrm{sca}}, \phi_{\mathrm{sca}}\right)-\ln P_{11, \mathrm{PGOM}}\left(\theta_{\text {sca }}\right.$, $\left.\phi_{\text {sca }}\right)$ for the $P_{11}$ element and $P_{i j, \mathrm{PGOM}}\left(\theta_{\text {sca }}, \phi_{\text {sca }}\right) / P_{11, \mathrm{PGOM}}$ $\left(\theta_{\text {sca }}, \phi_{\text {sca }}\right)-P_{i j, \text { IITM }}\left(\theta_{\text {sca }}, \phi_{\text {sca }}\right) / P_{11, \text { IITM }}\left(\theta_{\text {sca }}, \phi_{\text {sca }}\right)$ for other elements. Overall, the differences decrease as the size parameter increases.

As shown in Figs. 2 and 3, we find that the principle of the geometric optics is quite accurate for computing the near field at size parameter $\sim 120$ and more accurate for larger size parameters. Typically, a hexagonal plate or column tends to have a specific orientation when the maximum dimension of the particle exceeds $100 \mu \mathrm{m}$ (i.e., the size parameter is approximately 300 or larger for the three lidar wavelengths) based on aerodynamic simulations (Bréon and Dubrulle 2004; Hashino et al. 2016). Thus, it is feasible to use PGOM to compute the database for practical lidar simulations.

\section{b. The phase matrix of oriented ice crystals}

Figure 4 shows the two-dimensional phase matrix elements of HOPs with maximum dimension $100 \mu \mathrm{m}$ at wavelength $532 \mathrm{~nm}$ for various incident polar angles. The striking feature of $P_{11}$ of HOPs is the significant anisotropy with respect to the scattering polar angle. For $\theta_{\text {inc }}=0^{\circ}$, the scattering intensities in exact forward and backward directions are about $10^{4}$ and $10^{3}$, respectively, while the scattering intensity at any angle off the exact forward and backward directions is quite weak. As $\theta_{\text {inc }}$ increases, 
TABLE 1. General information about the single-scattering property database.

\begin{tabular}{|c|c|c|}
\hline Variables & Bins & Quantities \\
\hline Particle habits & 2 & HOP and HOC \\
\hline Wavelengths $\lambda$ & 3 & 355,532, and $1064 \mathrm{~nm}$ \\
\hline Maximum dimensions $D$ & 165 & $\begin{array}{l}50-10000 \mu \mathrm{m} \text { with same } \\
\text { intervals as in Y13 }\end{array}$ \\
\hline Incident polar angles $\theta_{\mathrm{inc}}$ & 94 & $\begin{array}{l}0^{\circ}-90^{\circ} \text { with inhomogeneous } \\
\text { intervals (see section } 2 \mathrm{c} \text { ) }\end{array}$ \\
\hline $\begin{array}{l}\text { Scattering polar } \\
\text { angles } \theta_{\text {sca }}\end{array}$ & 498 & $\begin{array}{l}0^{\circ}-180^{\circ} \text { with same intervals } \\
\text { as in Y13 }\end{array}$ \\
\hline $\begin{array}{l}\text { Scattering azimuthal } \\
\text { angles } \phi_{\text {sca }}\end{array}$ & 180 & $0^{\circ}-358^{\circ}$ with $2^{\circ}$ intervals \\
\hline
\end{tabular}

the forward scattering feature of $P_{11}$ changes slightly, and the backscattering peak occurs at $180^{\circ}-2 \theta_{\text {inc }}$ (Zhou et al. 2012). The other elements (i.e., $P_{12} / P_{11}$ and $P_{22} / P_{11}$ ) of the phase matrix increase their anisotropy with respect to the scattering polar and azimuth angles as $\theta_{\text {inc }}$ increases.

For $\theta_{\text {inc }}$ from $45^{\circ}$ to $75^{\circ}$, the phase matrix exhibits anisotropic features with respect to scattering polar and azimuth angles, and peaks corresponding to well-known optical phenomena associated with HOPs. In Fig. 4j, the scattering maxima appear at scattering polar angle $\sim 29^{\circ}$ and azimuth angle $\sim \pm 105^{\circ}$ corresponding to parhelia (Takano and Liou 1989; Forster et al. 2017), and the maxima approach scattering polar angle $22^{\circ}$ and scattering azimuth angle $\pm 90^{\circ}$ as $\theta_{\text {inc }}$ increases. In Figs. $4 \mathrm{~m}$ and $4 \mathrm{p}$, parabolic peaks seen at angles from the forward scattering direction with scattering azimuth angles $\pm 90^{\circ}$ to the backward scattering direction with scattering azimuth angles $\pm 180^{\circ}$ correspond to the parhelic circle (Liou and Yang 2016). In Fig. 4p, another parabolic peak appears at scattering polar angle $46^{\circ}$ and azimuth angles $\pm 180^{\circ}$, which provides the circumzenithal arc (Takano and Liou 1993), and the scattering peak is partially polarized as shown in Fig. 4q. For other elements of the phase matrix $\left(P_{12}\right.$ and $\left.P_{22}\right)$, the simulated results show many scattering maxima and minima and are generally complicated because of the contribution from multiple internal reflections and refractions. Note that very fine line-shaped scattering peaks might be artifacts caused by a limited number of orientations in the $\gamma$ rotational angle in which an infinite number of orientations is essentially required for accurate phase matrix computations.

Figure 5 is the same as Fig. 4 but shows the singlescattering properties of a HOC. The angular anisotropy of the two-dimensional phase matrix of a $\mathrm{HOC}$ is not as extreme as that of a HOP. In the case of $\theta_{\text {inc }}=0^{\circ}$ (Fig. 5a), $P_{11}$ shows scattering peaks at scattering polar

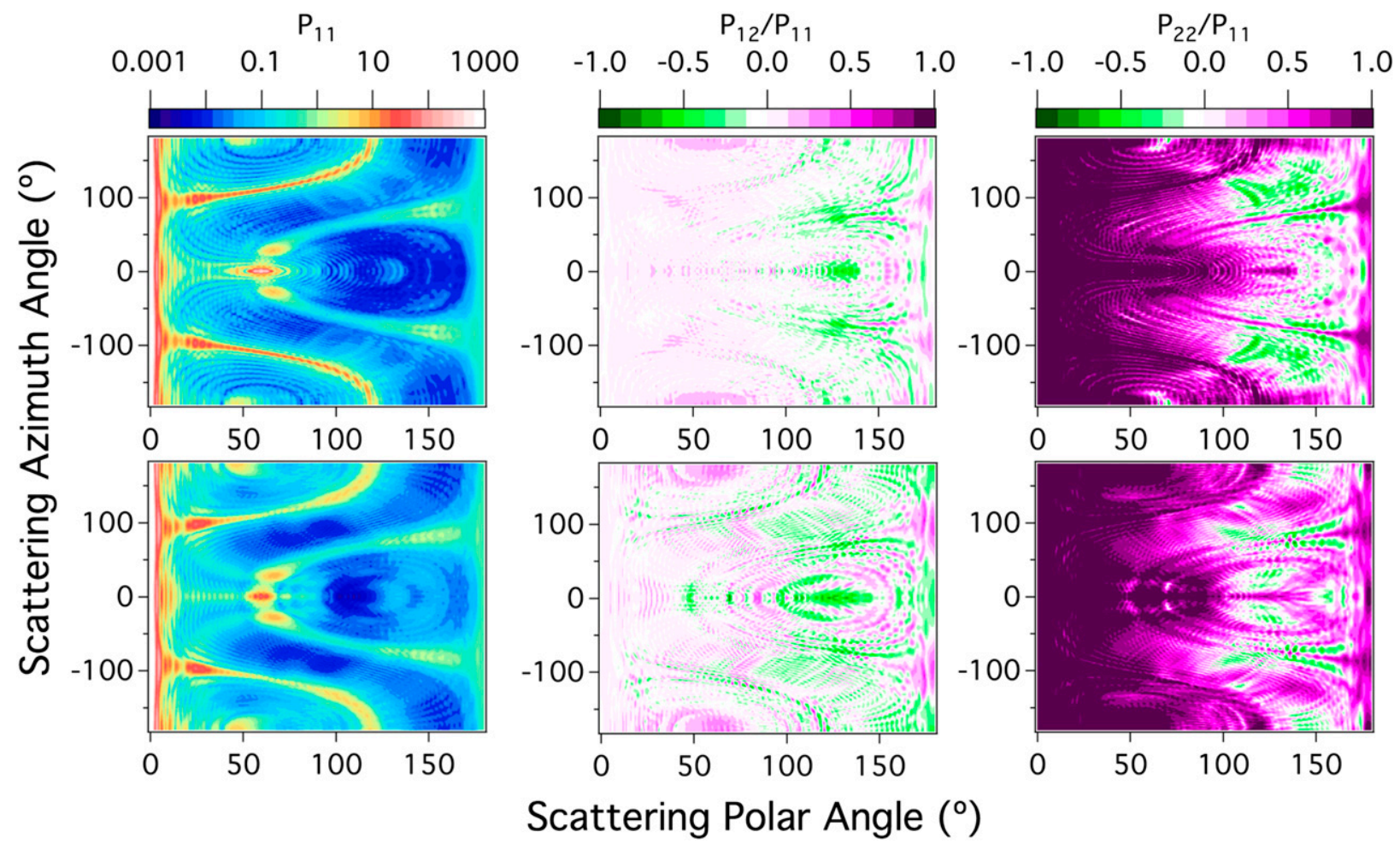

FIG. 2. Phase matrix elements (left) $P_{11}$, (middle) $P_{12} / P_{11}$, and (right) $P_{22} / P_{11}$ of a HOP computed with (top) PGOM and (bottom) IITM. The incident polar angle is $60^{\circ}$, and the size parameter $(\pi D / \lambda)$ is 120 based on $\lambda=532 \mathrm{~nm}$. 


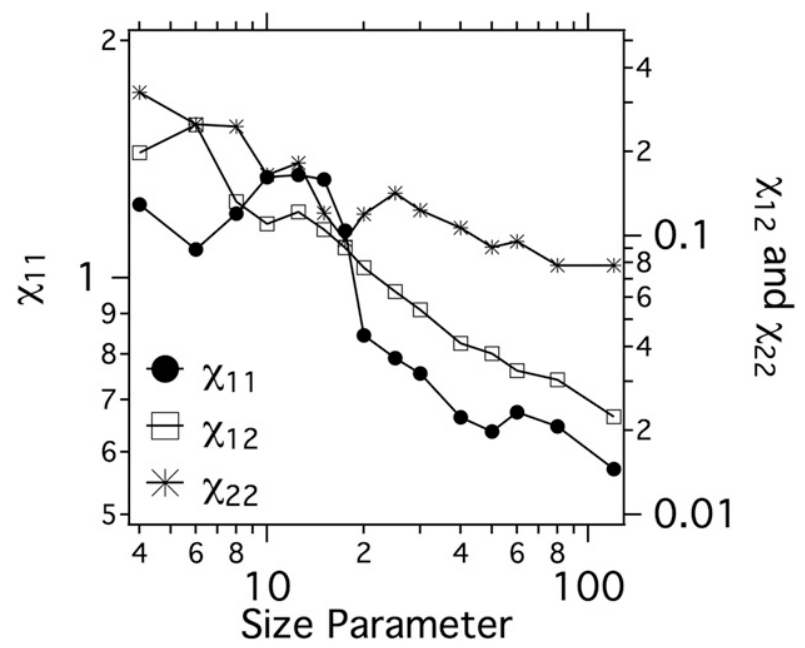

FIG. 3. Differences of the phase matrix elements $\left(P_{11}, P_{12} / P_{11}\right.$, and $\left.P_{22} / P_{11}\right)$ of a HOP between PGOM and IITM. The incident polar angle is $60^{\circ}$, and the size parameter $(\pi D / \lambda)$ ranges from 4 to 120 based on $\lambda=532 \mathrm{~nm}$.

angle $0^{\circ}$ due to diffraction, $22^{\circ}$ corresponding to $22^{\circ}$ halo features, and $180^{\circ}$ associated with specular reflection by the rectangular side faces of a HOC. The intensity of the backscattering peak of a HOC is smaller than the counterpart of a HOP by a factor of $\sim 10$. In the case of small $\theta_{\text {inc }}$ (Figs. 5d,g), the variation of the angular distribution of $P_{11}$ relative to an increase of $\theta_{\text {inc }}$ is limited except for backscattering peaks. In contrast, the angular distribution of $P_{22}$ in the backward scattering directions varies significantly as $\theta_{\text {inc }}$ increases (Figs. 5f,i).

For $\theta_{\text {inc }}$ from $45^{\circ}$ to $75^{\circ}$, the two-dimensional phase matrix shows a number of specific scattering peaks. Figure $5 \mathrm{j}$ shows a parabolic scattering peak associated with a parhelic circle, as in the case of a HOP. In addition, the circular scattering peak shifts from $22^{\circ}$ to a larger scattering polar angle depending on the scattering azimuth angle. This peak corresponds to a circumscribed halo, a specific optical phenomenon caused by a HOC under a certain range of solar zenith angle (Lynch and Livingston 2001). As $\theta_{\text {inc }}$ increases from $45^{\circ}$ (Figs. $5 \mathrm{~m}, \mathrm{p}$ ), the circular scattering peak is gradually separated into two major peaks, corresponding to the upper and lower tangent arcs. Moreover, weak scattering peaks with scattering polar angle $46^{\circ}$ correspond to a supralateral arc seen at scattering azimuth angles $>100^{\circ}$ and $<-100^{\circ}$ and infralateral arcs seen in the range of scattering azimuth angles from $\pm 50^{\circ}$ to $\pm 100^{\circ}$ (Liou and Yang 2016). These optical phenomena are not frequently observed (Sassen et al. 2003).

\section{c. Optical properties of oriented ice crystals}

Figure 6 shows two-dimensional plots of the extinction efficiency and asymmetry parameter of HOPs and
HOCs. The extinction efficiency of HOPs varies significantly with the incident polar angle and maximum dimension, which is consistent with previous studies (e.g., Bi et al. 2011). The oscillation of the extinction efficiency results from the interference between forward transmitted and diffracted light (Bi et al. 2010). In contrast, the variation of the extinction efficiency of a HOC is much smaller than that of a HOP. This is because the phase interference effect is suppressed because of the particle orientation of a HOC randomly rotated around two of the three axes (see section 2a) in contrast to that of a HOP randomly rotated around one of the three axes.

The asymmetry parameters of HOPs are generally around 0.9 for small incident polar angles except for HOPs in several size domains as shown in Fig. 6b, and those of HOCs vary between 0.75 and 0.95 depending on the incident polar angle and maximum dimension (Fig. 6d). In the size domains where the extinction efficiency of the HOP is low, the antiphase interference between transmitted and diffracted light is dominant. This results in a relatively weak forward scattering peak compared to the counterpart in the adjacent size domain, whereas the backscattering intensity due to the specular reflection by a HOP is not significantly different within the adjacent size domain. These conditions could cause significantly smaller asymmetry parameters, as displayed in Fig. 6b. Note that PGOM does not take account of the edge effect that contributes to the extinction of light by a particle ( $\mathrm{Bi}$ et al. 2010). The magnitude of the contribution of the edge effect to the single-scattering properties is relatively large for particles with low extinction efficiency values calculated with PGOM. Therefore, in these size domains where the extinction efficiency is low, the extinction efficiency might be underestimated, and therefore the asymmetry parameter could be uncertain.

Figure 7 shows the backscattering intensity of HOPs and HOCs with a maximum dimension of 100 and $1000 \mu \mathrm{m}$. The backscattering intensity becomes strongest when the incident polar angle is $0^{\circ}$, and it dramatically decreases as the incident polar angle slightly increases (Yang et al. 2003). The backscattering intensity of a HOP has oscillations with respect to the incident polar angle range, while that of a HOC smoothly decreases as the incident polar angle increases.

\section{Applications}

\section{a. Bulk optical properties of ice clouds containing} oriented ice crystals

To calculate realistic bulk optical properties of ice clouds containing oriented ice crystals, it is necessary to consider 1) the mixing ratio of oriented ice crystals, 

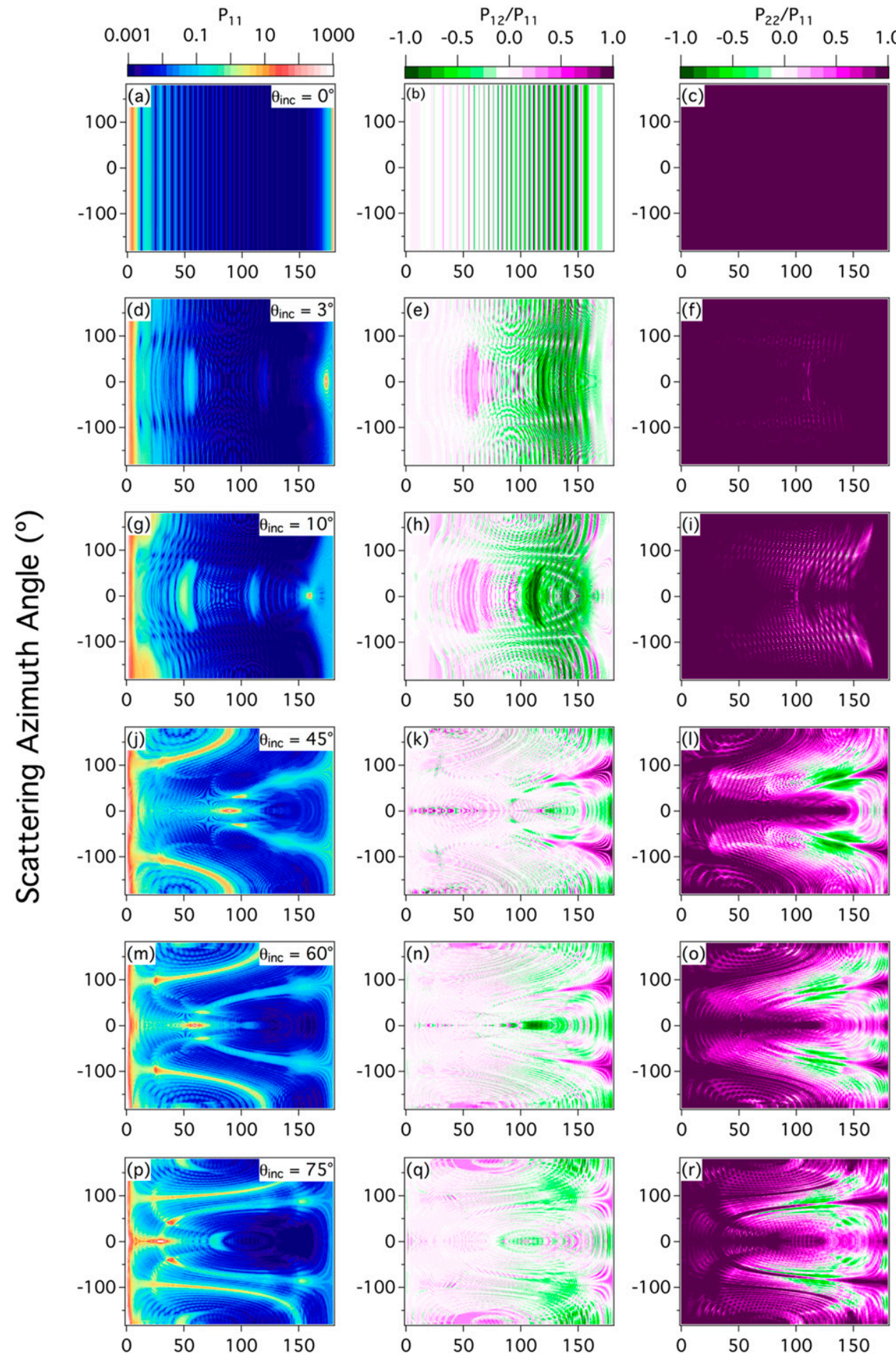

\section{Scattering Polar Angle $\left({ }^{\circ}\right)$}

FIG. 4. Phase matrix elements (left) $P_{11}$, (middle) $P_{12} / P_{11}$, and (right) $P_{22} / P_{11}$ of HOPs at $\lambda=532 \mathrm{~nm}$ with incident polar angles of (a)-(c) $0^{\circ}$, (d)-(f) $3^{\circ}$, (g)-(i) $10^{\circ}$, (j)-(l) $45^{\circ}$, (m) $-(\mathrm{o}) 60^{\circ}$, and (p) $-(\mathrm{r}) 75^{\circ}$. The maximum dimension is $100 \mu \mathrm{m}$. 

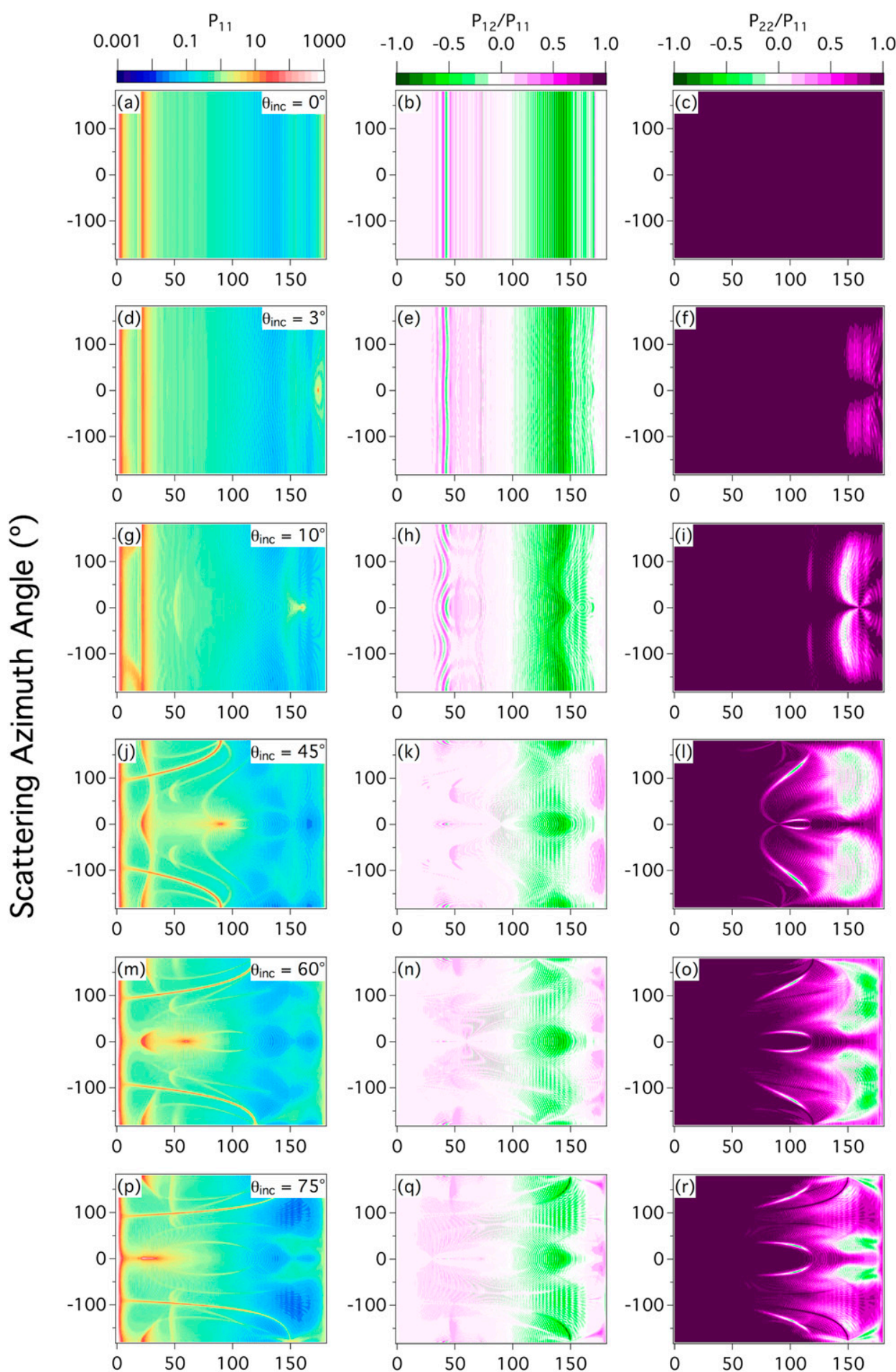

Scattering Polar Angle $\left({ }^{\circ}\right)$

FIG. 5. As in Fig. 4, but for HOCs. 


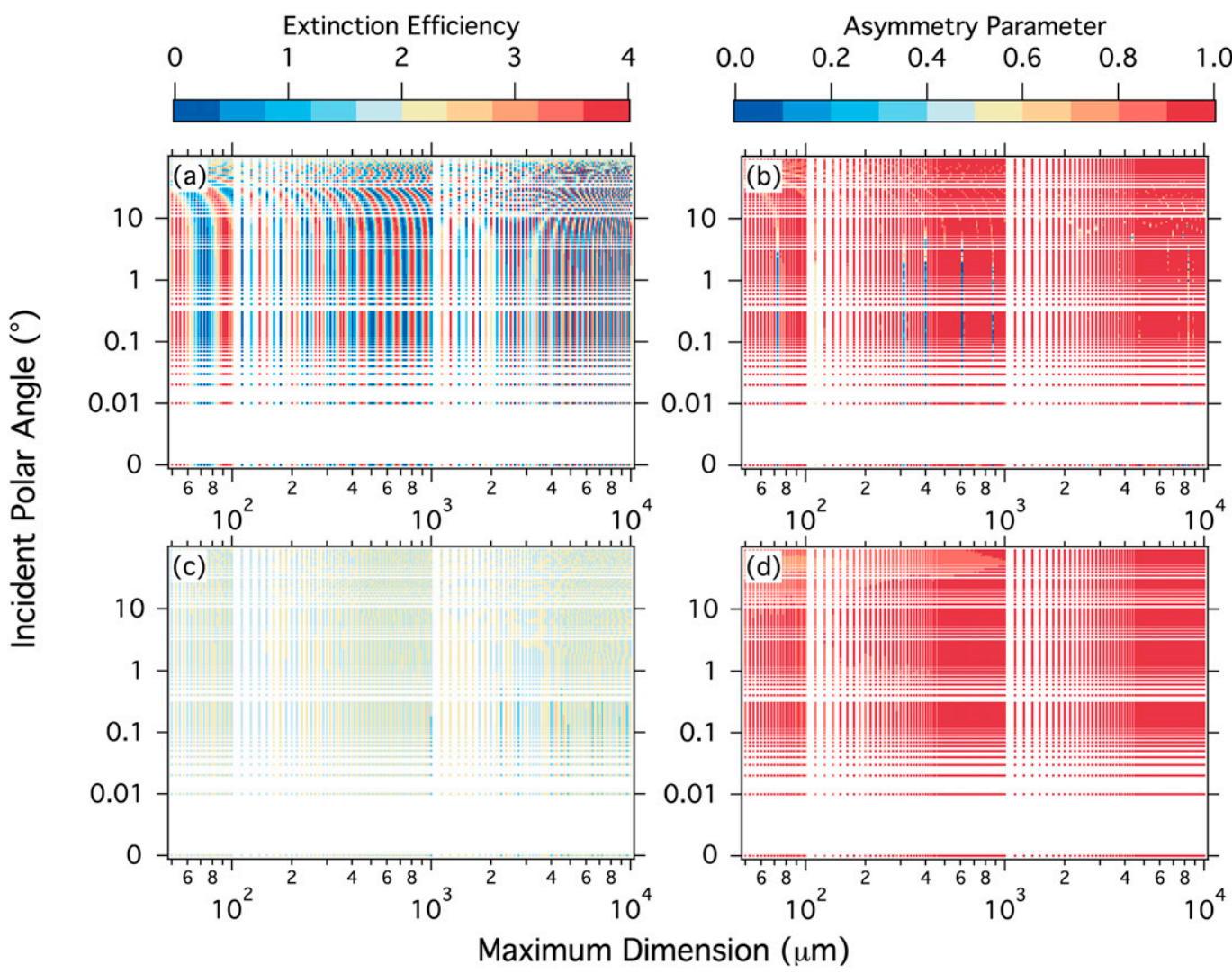

FIG. 6. The extinction efficiency and asymmetry parameter of (a),(b) HOPs and (c),(d) HOCs for various maximum dimensions and incident polar angles.

2) the size variation of oriented ice crystals, and 3) the tilting angle probability distribution of oriented ice crystals. Ice clouds often contain multiple ice particle shapes according to in situ measurements (e.g., Lawson et al. 2006), and irregular and aggregated ice crystals may not have a stable horizontal orientation. Therefore, this study assumes that the ice particle model consists of a mixture of hexagonal columns/plates and randomly oriented moderately roughened eight-column aggregate particles (Y13). Aerodynamic simulations of ice crystals demonstrate that a hexagonal particle can have a stable and horizontal orientation within the size range from a hundred to a few thousand micrometers under typical atmospheric conditions (Bréon and Dubrulle
2004; Hashino et al. 2014, 2016). At present, very little is known about the stability of particle orientations depending on its size and shape. Therefore, we assume that all hexagonal columns/plates have specific orientations with the maximum dimensions ranging from $D_{\mathrm{OIC}, \min }=$ $100 \mu \mathrm{m}$ to $D_{\text {OIC, } \max }=3000 \mu \mathrm{m}$, and have random orientations beyond the size range. Under realistic atmospheric conditions, hexagonal columns and plates may not be perfectly horizontally orientated but quasihorizontally orientated. Noel and Sassen (2005) found that the tilting angle $\theta_{\text {tilt }}$ of HOPs follows a Gaussian distribution based on ground-based scanning lidar observations. Therefore, we assume the probability density of the tilting angle $f\left(\theta_{\text {tilt }}, D\right)$ of HOP and HOC as

$$
f\left(\theta_{\text {tilt }}, D\right) d \phi_{\text {tilt }}=\left\{\begin{array}{cl}
\frac{1}{\pi\left\langle\Theta_{\text {tilt }}\right\rangle^{2}} \exp \left[-\left(\frac{\theta_{\text {tilt }}}{\left\langle\Theta_{\text {tilt }}\right\rangle}\right)^{2}\right] d \phi_{\text {tilt }}, & D \in\left[D_{\text {OIC,min }}, D_{\text {OIC,max }}\right] \\
U\left[0, \frac{\pi}{2}\right] d \phi_{\text {tilt }}, & \text { otherwise }
\end{array}\right.
$$

where $\left\langle\Theta_{\text {titt }}\right\rangle$ is the characterizing tilting angle (Bréon and Dubrulle 2004) corresponding to the standard deviation of the two-dimensional Gaussian distribution, and $U$ is a normalized uniform distribution over the $\theta_{\text {tilt }}$ and $\phi_{\text {tilt }}$ space. 


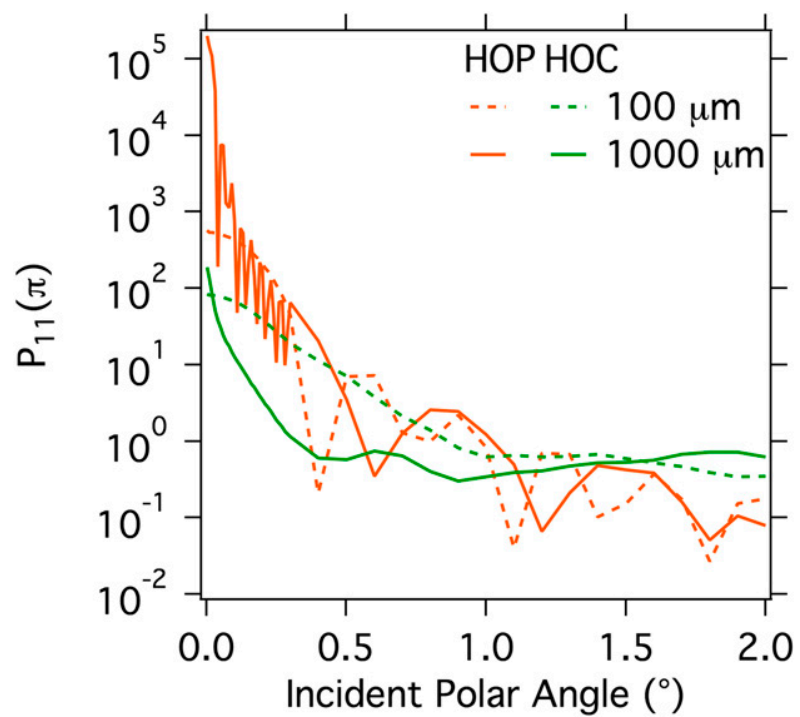

FIG. 7. The backscattering intensity of HOPs and HOCs. Solid and dashed lines indicate maximum dimensions of 100 and $1000 \mu \mathrm{m}$, respectively. Orange and green colors show HOPs and HOCs.

Figure 8 illustrates the ice model containing HOPs as an example. The ice model containing HOCs is similar

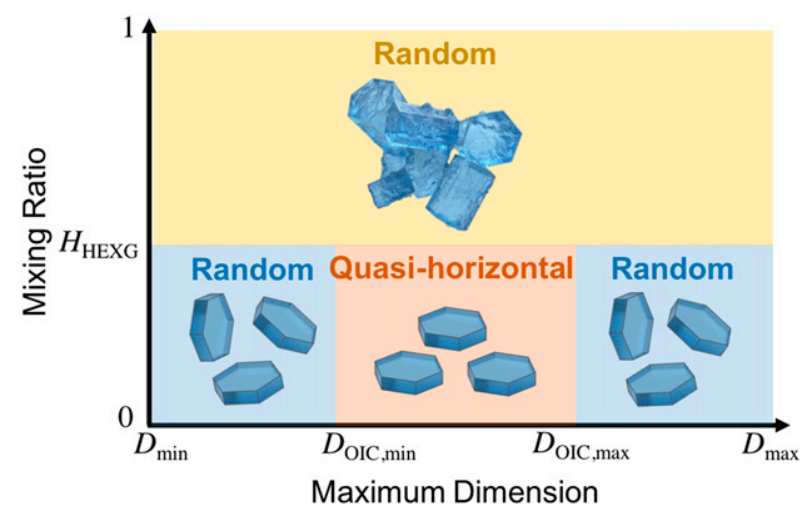

FIG. 8. A diagram of bulk ice model involving oriented ice crystals (showing HOPs).

to Fig. 8 except for columns. To calculate the bulk optical properties of ice clouds containing oriented ice crystals, the geometric and single-scattering properties of oriented ice crystals are weighted with $f\left(\theta_{\text {tilt }}, D\right)$, which is close to a delta function due to a small $\left\langle\Theta_{\text {tilt }}\right\rangle$. Therefore, the orientation-weighted single-scattering properties (indicated with subscript "ori”) are

$$
\mathbf{P}_{\text {ori }}\left(\theta_{\text {sca }}, \phi_{\text {sca }}, \theta_{\text {inc }}, D\right)=\frac{\int_{0}^{\pi / 2} \int_{0}^{\pi} f\left(\theta_{\text {tilt }}, D\right) P\left(\theta_{\text {sca }}, \phi_{\text {sca }}, \theta_{\text {inc }}^{*}, D\right) \theta_{\text {tilt }} d \phi_{\text {tilt }} d \theta_{\text {tilt }}}{\int_{0}^{\pi / 2} \int_{0}^{\pi} f\left(\theta_{\text {tilt }}, D\right) \theta_{\text {tilt }} d \phi_{\text {tilt }} d \theta_{\text {tilt }}},
$$

$$
X_{\text {ori }}\left(\theta_{\text {inc }}, D\right)=\frac{\int_{0}^{\pi / 2} \int_{0}^{\pi} f\left(\theta_{\text {tilt }}, D\right) X\left(\theta_{\text {inc }}^{*}, D\right) \theta_{\text {tilt }} d \phi_{\text {tilt }} d \theta_{\text {tilt }}}{\int_{0}^{\pi / 2} \int_{0}^{\pi} f\left(\theta_{\text {tilt }}, D\right) \theta_{\text {tilt }} d \phi_{\text {tilt }} d \theta_{\text {tilt }}}
$$

where $X$ includes the extinction cross section $\sigma_{\text {ext }}$, single-scattering albedo $\omega$, and asymmetry parameter $g$, and

$$
\cos \theta_{\text {inc }}^{*}=\cos \theta_{\text {inc }} \cos \theta_{\text {tilt }}+\sin \theta_{\text {inc }} \sin \theta_{\text {tilt }} \cos \phi_{\text {tilt }} .
$$

The particle size distribution (PSD) $n(D)$ of the bulk ice model is assumed to be the gamma distribution parameterized by Iwabuchi et al. (2012). The following equations omit the subscript "ori" for simplicity. The bulk optical properties of ice clouds are obtained from PSD, the mixing ratio in terms of number $H_{i}$, the orientation-weighted single-scattering properties of hexagonal columns/plates (subscript "HEXG"), and the single-scattering properties of randomly oriented eight-column aggregate particles (subscript "CAGG”), with the following equations:

$$
\left\langle\sigma_{\text {ext }}\left(\theta_{\mathrm{inc}}\right)\right\rangle=\int_{D_{\min }}^{D_{\max }} \sum_{i} H_{i} \sigma_{\mathrm{ext}, i} n(D) d D,
$$

$$
\left\langle\omega\left(\theta_{\mathrm{inc}}\right)\right\rangle=\frac{\int_{D_{\min }}^{D_{\max }} \sum_{i} H_{i} \omega_{i} \sigma_{\mathrm{ext}, i} n(D) d D}{\left\langle\sigma_{\mathrm{ext}}\left(\theta_{\mathrm{inc}}\right)\right\rangle},
$$

$$
\left\langle g\left(\theta_{\text {inc }}\right)\right\rangle=\frac{\int_{D_{\min }}^{D_{\max }} \sum_{i} H_{i} g_{i} \omega_{i} \sigma_{\text {ext }, i} n(D) d D}{\left\langle\omega\left(\theta_{\text {inc }}\right)\right\rangle\left\langle\sigma_{\text {ext }}\left(\theta_{\text {inc }}\right)\right\rangle},
$$

$\left\langle\mathbf{P}\left(\theta_{\text {sca }}, \phi_{\text {sca }}, \theta_{\text {inc }}\right)\right\rangle=\frac{\int_{D_{\text {min }}}^{D_{\text {max }}} \sum_{i} H_{i} \mathbf{P}_{i} \omega_{i} \mathbf{K}_{\text {ext }, i} n(D) d D}{\left\langle\omega\left(\theta_{\text {inc }}\right)\right\rangle\left\langle\sigma_{\text {ext }}\left(\theta_{\text {inc }}\right)\right\rangle}$,

where $X$ is a function of $D$ for CAGG and a function of both $D$ and $\theta_{\text {inc }}$ for HEXG, and the mixing ratios satisfy $H_{\mathrm{HEXG}}+H_{\mathrm{CAGG}}=1$. As the bulk ice model contains 
oriented crystals, the $4 \times 4$ extinction matrix $\mathbf{K}_{\text {ext }}$ for HEXG has nonzero values in the off-diagonal elements (Mishchenko 1991; Zhou et al. 2012) as

$\mathbf{K}_{\mathrm{ext}}\left(\theta_{\mathrm{inc}}, D\right)=\sigma_{\mathrm{ext}}\left(\theta_{\mathrm{inc}}, D\right)\left[\begin{array}{cccc}1 & Q_{\mathrm{pol}} & 0 & 0 \\ Q_{\mathrm{pol}} & 1 & 0 & 0 \\ 0 & 0 & 1 & Q_{\mathrm{cpol}} \\ 0 & 0 & Q_{\mathrm{cpol}} & 1\end{array}\right]$,

where $Q_{\text {pol }}$ and $Q_{\text {cpol }}$ are the ratios of the extinction coefficient in terms of linear and circular polarized light to $\sigma_{\text {ext }}$. A detailed description of these quantities is given in appendix A. Because these ratios are negligibly small, we omit these off-diagonal elements. Therefore, Eq. (11) becomes

$\left\langle\mathbf{P}\left(\theta_{\text {sca }}, \phi_{\text {sca }}, \theta_{\text {inc }}\right)\right\rangle=\frac{\int_{D_{\min }}^{D_{\max }} \sum_{i} H_{i}\left\langle\mathbf{P}_{i}\right\rangle \omega_{i} \sigma_{\text {ext }, i} n(D) d D}{\left\langle\omega\left(\theta_{\text {inc }}\right)\right\rangle\left\langle\sigma_{\text {ext }}\left(\theta_{\text {inc }}\right)\right\rangle}$.

Figure 9 shows the lidar ratio $\left[4 \pi / \omega P_{11}(\pi)\right]$ of the bulk ice model containing HOPs and HOCs in the case of incident polar angle of $0.3^{\circ}$. The lidar ratio varies with both the fraction of oriented ice crystals and the characterizing tilting angle. When the characterizing tilting angle and fraction of oriented ice crystals are the same between HOPs and HOCs, the lidar ratio of ice clouds containing HOPs is smaller than that of HOCs. This is due to stronger backscattering intensity of HOPs than that of HOCs (Fig. 7). In Fig. 9a, the lidar ratio can be lower than 1 when the HOP fraction is large, and the characterizing tilting angle is small. Although such low lidar ratios in ice clouds are rarely observed (Saito et al. 2017), the results indicate that low lidar ratios are mainly caused by HOPs. However, as shown in Fig. 9b, a lidar ratio greater than 1 can be explained by both HOPs and HOCs, and a mixture of these particles. Saito et al. (2017) show that the lidar ratio of cirrus clouds over the global ocean varies from 20 to $50 \mathrm{sr}$. The range of the lidar ratio corresponds to the fraction of HOPs $0.1 \%-0.5 \%$ and fraction of HOCs $2 \%-10 \%$ at maximum depending on the characterizing tilting angle. This implies that estimations of the fractions of oriented ice crystals are quite sensitive to an assumption of the particle orientation and habit.

\section{b. Lidar simulations}

Lidar measurements of the integrated attenuated backscattering (IAB) $\gamma^{\prime}$ and volume depolarization
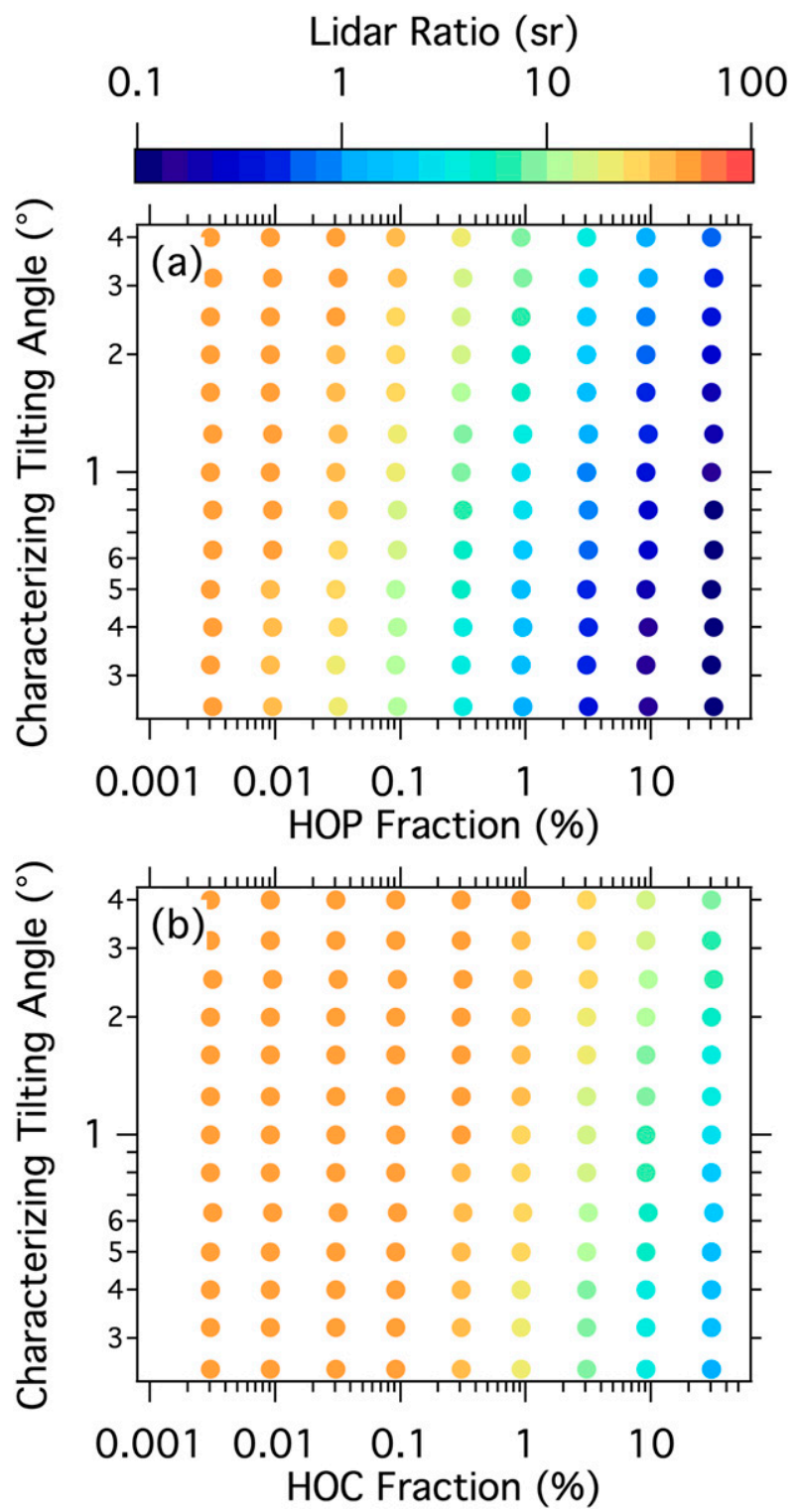

FIG. 9. The bulk lidar ratio of ice clouds involving (a) HOPs and (b) HOCs with respect to various characterizing tilting angles and the fraction of HOPs and HOCs. The incident polar angle is $0.3^{\circ}$.

ratio $\delta$ of ice clouds are useful to diagnose the cloud phase (Hu et al. 2009) and to investigate particle shapes in ice clouds (Sassen 1991; Yoshida et al. 2010; Saito et al. 2017). The Cloud-Aerosol Lidar with Orthogonal Polarization (CALIOP) onboard the CloudAerosol Lidar and Infrared Pathfinder Satellite Observations (CALIPSO; Winker et al. 2009) has provided unprecedented observations to study the optical and microphysical properties of ice clouds on a global scale. At the initial stage, the CALIOP sensor pointed in a quasi-nadir direction [off-nadir pointing angle $\left.\theta_{\text {off }}=0.3^{\circ}\right]$. In November 2007, CALIOP 
changed the pointing direction to $\theta_{\text {off }}=3^{\circ}$ to avoid specular reflections from horizontally oriented ice crystals. Therefore, global lidar measurements based on both quasi-nadir and off-nadir pointing angles are available, which provides a unique opportunity to study oriented ice crystals in ice clouds.

To demonstrate the expected differences in lidar measurements between the two pointing configurations, we simulate lidar signals from ice clouds containing horizontally oriented ice crystals based on the aforesaid bulk optical properties of ice clouds. We use the Monte Carlo radiative transfer model ( $\mathrm{Hu}$ et al. 2001), which has been modified to account for oriented ice crystals (Zhou et al. 2012).

Figure 10 shows IAB and the volume depolarization ratio of ice clouds containing HOPs and HOCs as a function of the fraction of oriented ice crystals for both quasi-nadir and off-nadir pointing angle cases. These lidar signals are sensitive to the characterizing tilting angle and the fraction of HOPs and HOCs. In Fig. 10a, a higher HOP fraction causes a larger IAB and a lower volume depolarization ratio. In addition, the impact of the variation of the characterizing tilting angle from $0.4^{\circ}$ to $2.5^{\circ}$ on lidar signals is as large as the variation of the HOP fraction within an order of magnitude. With an offnadir pointing angle of $3^{\circ}$ (Fig. 10b), lidar signals are much less sensitive to HOPs because of avoiding their specular reflection. However, the volume depolarization ratio becomes smaller when the HOP fraction $>0.1 \%$ for $\left\langle\Theta_{\text {tilt }}\right\rangle=2.5^{\circ}$ and the HOP fraction $>1 \%$ for $\left\langle\Theta_{\text {tilt }}\right\rangle=1^{\circ}$ and $0.4^{\circ}$. The CALIOP measurements with an off-nadir pointing angle of $3^{\circ}$ do not show particular characteristics in IAB and the volume depolarization ratio because of oriented ice crystals (Zhou et al. 2012). By comparing the lidar simulations with the CALIOP measurements, our simulations indicate that the maximum fraction of HOPs in ice clouds may be less than $\sim 1 \%$ under the assumption that oriented ice crystals are all hexagonal plates.
The variation of the lidar signals of ice clouds containing HOCs is similar to that of HOPs (Figs. 10c,d). However, in the case of an off-nadir pointing angle of $0.3^{\circ}$, the fraction of oriented ice crystals where the volume depolarization ratio starts decreasing is larger for HOCs than that of HOPs by one to two orders of magnitude of the fraction. This is primarily due to the backscattering intensity of HOCs weaker than that of HOPs (Fig. 7). Most studies regarding oriented ice crystals take into account the fraction of perfectly horizontally oriented plates (e.g., Okamoto et al. 2010). However, this study demonstrates the importance of the characterizing tilting angle and habit (i.e., HOPs or HOCs) of oriented ice crystals on lidar simulations.

\section{c. Optical phenomena simulations}

As described in section $3 b$, oriented ice crystals cause several optical phenomena under optically thin ice cloud conditions. The rarity of these optical phenomena seen from the ground depends on the atmospheric turbulence state, ice particle shape, and scattering intensity of ice particles corresponding to the optical phenomena. Pattloch and Tränkle (1984) performed monochromatic optical phenomena simulations based on the Monte Carlo method using the phase function of ice crystals computed with GCOM. This study improves the simulation of the optical phenomena caused by oriented ice crystals using the ice bulk optical properties and an optical phenomenon simulator.

The optical phenomenon simulator includes a vector radiative transfer model based on the singlescattering approximation and a mapping formula for converting directional radiance from the laboratory coordinate to an image coordinate (see appendix B). We calculate sky radiance $\left[\mathbf{I}=(I, Q, U, V)^{\mathrm{T}}\right]$ at the ground at every viewing zenith angle $\theta$ and an azimuth angle $\phi$ based on a given solar direction [the solar zenith and azimuth angles $\theta_{o}$ and $\phi_{o}$ ] and irradiance $\left[\mathbf{E}_{o}=\left(F_{o}, 0,0,0\right)^{\mathrm{T}}\right]$ as

$$
\mathbf{I}(\theta, \phi)=\mathbf{E}_{o}\left|\cos \theta_{o}\right| \exp \left[-\bar{\tau}\left(\theta_{o}\right) /\left|\cos \theta_{o}\right|\right] \frac{\bar{\omega} \mathbf{\mathbf { P }}}{4 \pi} \frac{1-\exp \left[-\bar{\tau}\left(\theta_{o}\right) /\left(1 /\left|\cos \theta_{o}\right|+1 / \cos \theta\right)\right]}{\left|\cos \theta_{o}\right|+\cos \theta}
$$

where the optical thickness $\bar{\tau}$, single-scattering albedo $\bar{\omega}$, and phase matrix $\overline{\mathbf{P}}$ of the scattering layer that includes molecules and ice crystals are given by

$$
\begin{aligned}
& \bar{\tau}\left(\theta_{o}\right)=\tau_{R}+\tau_{C}\left(\theta_{o}\right), \\
& \bar{\omega}\left(\theta_{o}\right)=\frac{\tau_{R}+\tau_{C}\left(\theta_{o}\right)\left\langle\omega\left(\theta_{o}\right)\right\rangle}{\tau_{R}+\tau_{C}\left(\theta_{o}\right)},
\end{aligned}
$$

$$
\begin{aligned}
& \overline{\mathbf{P}}\left(\theta_{\text {sca }}, \phi_{\text {sca }}, \theta_{o}\right) \\
& \quad=\frac{\tau_{R} \mathbf{P}_{R}\left(\theta_{\text {sca }}\right)+\tau_{C}\left(\theta_{o}\right)\left\langle\omega\left(\theta_{o}\right)\right\rangle\left\langle\mathbf{P}\left(\theta_{\text {sca }}, \phi_{\text {sca }}, \theta_{o}\right)\right\rangle}{\tau_{R}+\tau_{C}\left(\theta_{o}\right)\left\langle\omega\left(\theta_{o}\right)\right\rangle},
\end{aligned}
$$

where $\mathbf{P}_{R}$ is the phase matrix of Rayleigh scattering. The optical thickness of molecules is parameterized as 

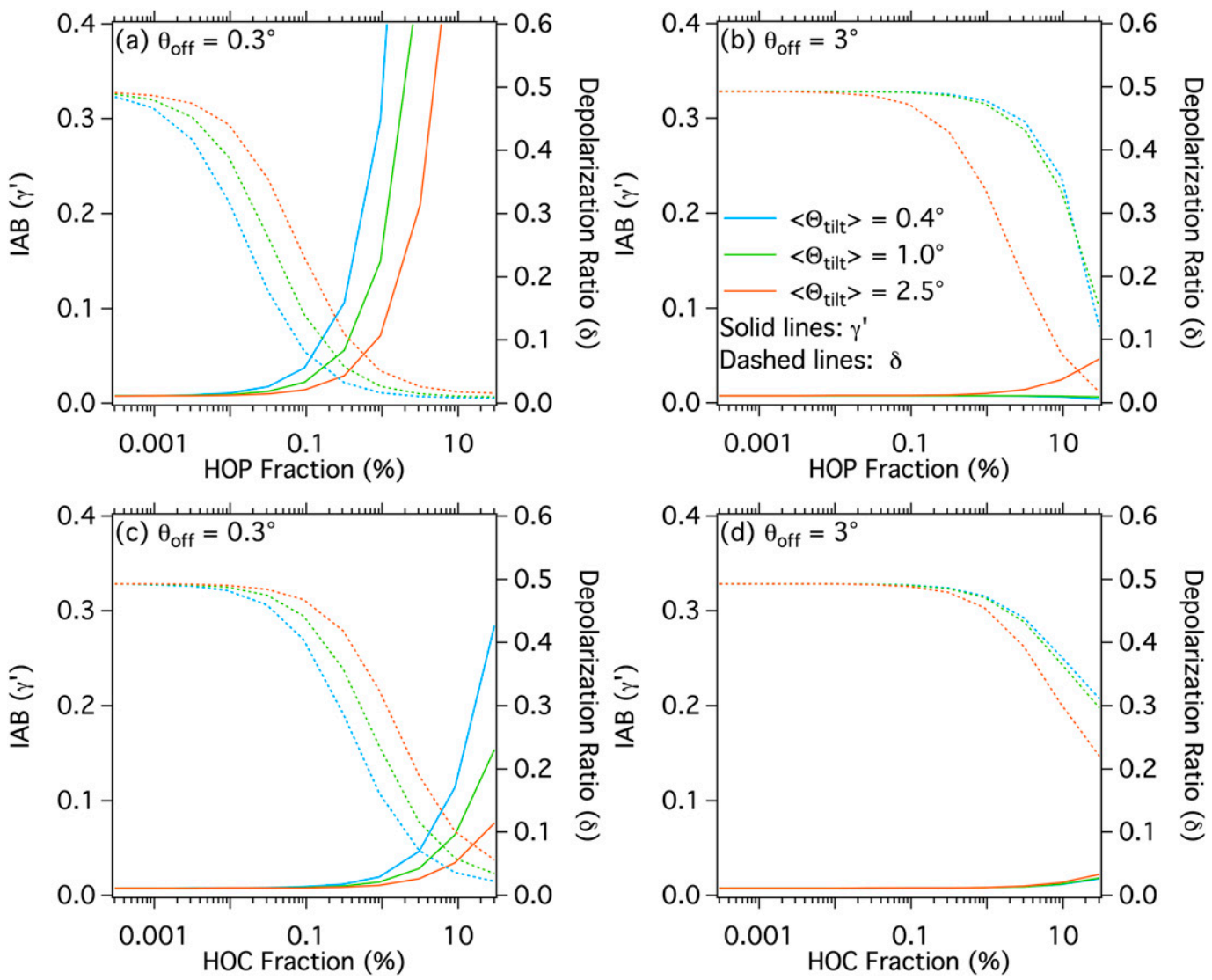

FIG. 10. Simulations of lidar signals from ice clouds involving (a),(b) HOPs and (c),(d) HOCs for the off-nadir angles of (left) $0.3^{\circ}$ and (right) $3^{\circ}$. Solid and dashed lines indicate IAB and the volume depolarization ratio, respectively. Colors indicate the characterizing tilting angle of oriented ice crystals.

$\tau_{R}(\lambda)=0.00864 \lambda^{-(3.916+0.074 \lambda+0.05 / \lambda)}$, where $\lambda$ is in micrometers (Fröhlich and Shaw 1980). The cloud optical thickness $\left[\tau_{C}\left(\theta_{o}\right)=\mathrm{GN}_{C}\left\langle\sigma_{\text {ext }}\left(\theta_{o}\right)\right\rangle\right]$ is calculated from the cloud geometric thickness $(G=1 \mathrm{~km})$, the number of ice crystals $N_{C}$ and the bulk extinction cross section.

In the conversion to the image coordinate system, we assume an equisolid-angle fish-eye lens with half angle of view $90^{\circ}$. The mapping formulas are given in appendix $B$. The radiative transfer calculations are performed for wavelengths $425 \mathrm{~nm}$ for the blue channel, $525 \mathrm{~nm}$ for the green channel, and $625 \mathrm{~nm}$ for the red channel. (B, G, and R channels, respectively). Since the bulk ice optical properties are available only for the three lidar wavelengths, we use the bulk ice optical properties at 355, 532, and $1064 \mathrm{~nm}$ for $\mathrm{B}, \mathrm{G}$, and R channels to simulate each "pseudo" monochromatic photographic image. Finally, the radiance computed in each monochromatic image is discretized as an eight-bit signal, and we compose the three monochromatic images into a "pseudo" color image. Note that the color difference due to the refraction of ice crystals is enhanced because the difference of the real part of the ice refractive index between 355 and $1064 \mathrm{~nm}$ (the shortest and longest lidar wavelengths) are larger than that between 425 and $625 \mathrm{~nm}$ (B and R channels).

Figure 11 shows simulated pseudophotographic images of optical phenomena caused by HOPs and HOCs with solar zenith angles of $35^{\circ}$ and $70^{\circ}$. The cloud optical thickness and the cloud-particle effective radius are assumed to be 0.3 and $30 \mu \mathrm{m}$ for each simulation. The mixing ratios of hexagonal plates (Figs. 11a,b) and columns (Figs. 11c,d) are 0.3 and 0.2 , respectively. Therefore, the total fractions of oriented ice crystals are $\sim 9 \%$ for HOPs and $\sim 6 \%$ for HOCs. Overall, all cases show a bright $22^{\circ}$ halo, which are caused by randomly oriented hexagonal ice crystals. Moreover, some rare optical phenomena such as an infralateral arc (Fig. 11c) and a supralateral arc (Fig. 11d) appear (Pattloch and Tränkle 1984). With a solar zenith angle of $35^{\circ}$ (Fig. 11a), the sky displays a dim circumhorizontal arc near the horizon and both parhelic circle and parhelia beside the sun. These optical phenomena 

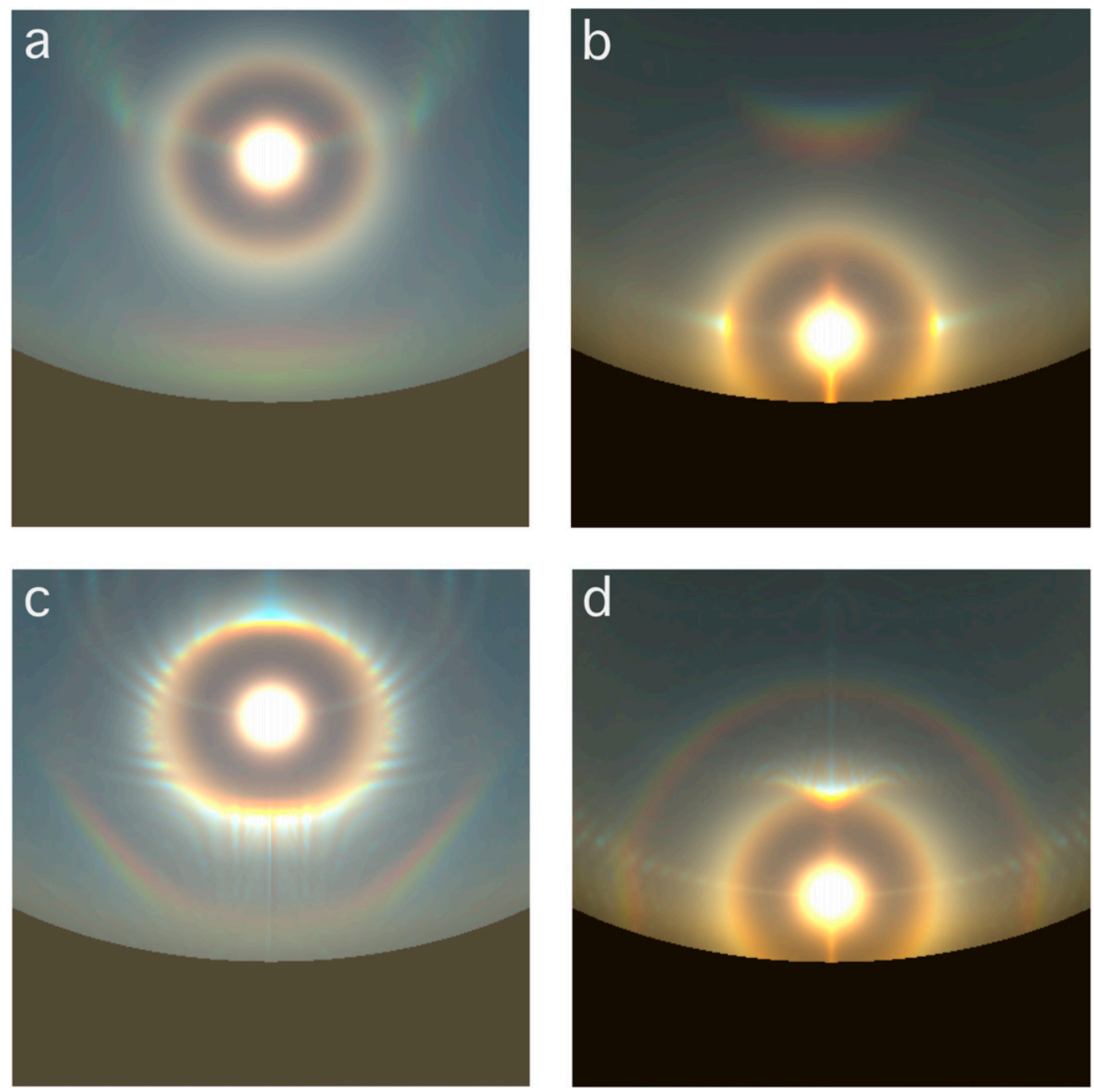

FIG. 11. Pseudophotographic images of optical phenomena caused by (a),(b) HOPs and (c),(d) HOCs. The solar zenith angles are (left) $35^{\circ}$ and (right) $70^{\circ}$.

are caused by HOPs. With a solar zenith angle of $70^{\circ}$ (Fig. 11b), parhelia become much brighter as indicated in Figs. $4 \mathrm{j}$ and $4 \mathrm{~m}$. In addition, a dim circumzenithal arc appears at approximately $46^{\circ}$ above the solar direction. In Fig. 11c, a bright circumscribed halo is seen, which is caused by HOCs. Note that the striping feature in the circumscribed halo is an artifact caused by the limited number of orientations of HOCs. When the solar zenith angle is large, the circumscribed halo is separated into an upper tangent arc that appears at $22^{\circ}$ above the solar direction (Fig. 11d) and a lower tangent arc that would be seen below the horizon (Greenler and Mallmann 1972). Note that the contribution of multiple scattering to radiance becomes more substantial as cloud optical thickness and solar zenith angle increase. Therefore, simulated radiance could be underestimated, and the radiance distribution might be smoother.

It is interesting to simulate optical phenomena images with a linear polarized filter. Figure 12 shows a simulated photographic image of optical phenomena with an idealized linear polarized filter (Voss and Liu 1997). The circumzenithal arc simulated with the linear polarized filter parallel to the scattering plane (right half of Fig. 12) is brighter than the counterpart with the filter perpendicular to the scattering plane (left half of Fig. 12). Rayleigh scattering intensity is larger with the filter perpendicular to the scattering plane compared to the counterpart with the filter parallel to the scattering plane. This opposite feature is caused by positive $P_{12}$ values of HOPs at corresponding scattering maxima (Figs. 4p,q) and negative $P_{12}$ values of Rayleigh scattering. 


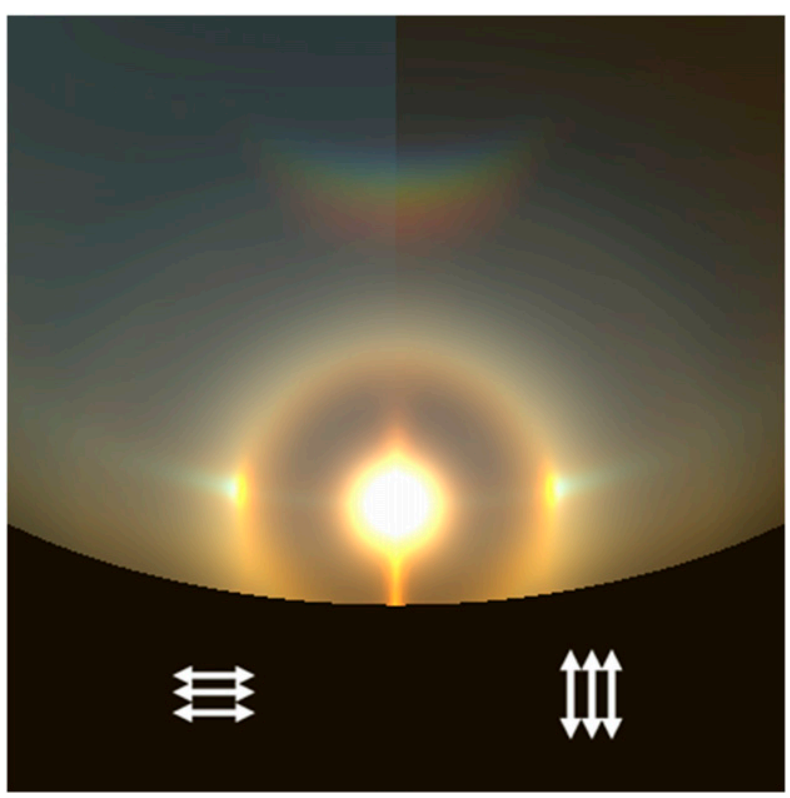

FIG. 12. As in Fig. 11b, but for the simulation with a linear polarized filter that transmits electromagnetic waves (left) perpendicular and (right) parallel to the scattering plane.

\section{Summary}

A database of the single-scattering properties of oriented ice crystals (TAMUoic2019) was developed with the physical-geometric optics method (PGOM). The database includes the single-scattering properties of horizontally oriented hexagonal plates (HOPs) and horizontally oriented hexagonal columns (HOCs) at the three lidar wavelengths $(355,532$, and $1064 \mathrm{~nm})$ for particle sizes from 50 to $10000 \mu \mathrm{m}$ and the incident polar angles from $0^{\circ}$ to $90^{\circ}$. The two-dimensional phase matrices computed with PGOM are consistent with the solutions based on the exact invariant-imbedding T-matrix method (IITM). The two-dimensional phase matrices of HOPs and HOCs show significant angular anisotropy with respect to both scattering polar and azimuth angles, and display intriguing scattering maxima correspond to specific optical phenomena. These scattering patterns vary with the incident polar angle. The extinction efficiency of HOCs is close to 2, whereas that of HOPs has significant oscillations with respect to the maximum dimension and incident polar angle. The backscattering intensities of HOPs and HOCs are sensitive to the incident polar angle.

The bulk optical properties of ice clouds containing quasi-horizontally oriented ice crystals are calculated from the TAMUoic2019 database. We took into account the mixture of the eight-column aggregates and hexagonal ice crystals, the size dependence of the hexagonal particle orientation, and the characterizing tilting angle of oriented ice crystals (i.e., HOPs and HOCs). Simulated lidar ratios of ice clouds vary from 0.1 to $50 \mathrm{sr}$ for HOPs and 2 to $50 \mathrm{sr}$ for HOCs. The lidar simulation is performed with the aforesaid bulk optical properties. As the fraction of quasi-horizontally oriented ice crystals in ice clouds increases, IAB increases, and volume depolarization ratio decreases. However, the sensitivity of lidar signals to the HOP fraction is $10-20$ times larger than that of the HOC fraction. The present optical phenomena simulations show parhelia, and circumhorizontal and circumzenithal arcs for ice clouds containing HOPs, a circumscribed halo and an upper tangent arc for ice clouds containing HOCs, and a parhelic circle for both cases. These simulated optical phenomena are consistent with photographic observations, which indicate the robustness of the phase matrices in the TAMUoic2019 database.

The TAMUoic2019 database could be a useful resource for radiative transfer simulations and remote sensing implementations involving ice clouds. In particular, this will contribute to improving ice cloud remote sensing with ground-based and spaceborne lidar measurements. CALIOP has provided backscattering measurements of global ice clouds over a decade. Similarly, future Earth Clouds, Aerosols and Radiation Explorer (EarthCARE) mission (Illingworth et al. 2015) will provide the backscattering measurements. Furthermore, measurements of optical phenomena by ground-based cameras are useful for characterizing ice crystal shape (Forster et al. 2017). The TAMUoic2019 database in conjunction with an optical phenomenon simulator may potentially contribute to quantifying the characteristics of the ice crystal shape.

Acknowledgments. This research was supported by the National Science Foundation (Grant AGS-1826936) and partly by the endowment funds related to the David Bullock Harris Chair in Geosciences at the College of Geosciences, Texas A\&M University (Grant 02-5122311000). The present numerical computations were conducted at the Texas A\&M Supercomputing Facilities, and the authors gratefully acknowledge facility staff for their help and assistance. The authors thank Dr. Hironobu Iwabuchi for a major contribution to the development of a sky image simulator. The TAMUoic2019 database is publicly available upon a request to the authors.

\section{APPENDIX A}

\section{The Extinction Matrix of Ice Clouds Involving Oriented Ice Crystals}

The forward-scattering amplitude matrix $\mathbf{S}\left(\theta_{\text {inc }}, D\right)$ provides the extinction cross sections as 


$$
\begin{gathered}
\sigma_{\text {ext }}\left(\theta_{\text {inc }}, D\right)=\frac{2 \pi}{k^{2}} \operatorname{Re}\left[\mathbf{S}_{11}\left(\theta_{\text {inc }}, D\right)+\mathbf{S}_{22}\left(\theta_{\text {inc }}, D\right)\right], \\
\sigma_{\text {pol }}\left(\theta_{\text {inc }}, D\right)=\frac{2 \pi}{k^{2}} \operatorname{Re}\left[\mathbf{S}_{11}\left(\theta_{\text {inc }}, D\right)-\mathbf{S}_{22}\left(\theta_{\text {inc }}, D\right)\right], \\
\sigma_{\text {cpol }}\left(\theta_{\text {inc }}, D\right)=\frac{2 \pi}{k^{2}} \operatorname{Im}\left[\mathbf{S}_{11}\left(\theta_{\text {inc }}, D\right)-\mathbf{S}_{22}\left(\theta_{\text {inc }}, D\right)\right],
\end{gathered}
$$

where $k$ is the wavenumber in free space; and $\mathrm{Re}$ and $\mathrm{Im}$ denote the real and imaginary parts, respectively (Zhou et al. 2012). The extinction cross sections are weighted with $f\left(\theta_{\text {tilt }}, D\right)$ similarly to Eq. (6b), and we obtain $\sigma_{\text {ext }}\left(\theta_{\text {inc }}, D\right), \sigma_{\text {pol }}\left(\theta_{\text {inc }}, D\right)$, and $\sigma_{\text {cpol }}\left(\theta_{\text {inc }}, D\right)$. Therefore, the extinction matrix $\mathbf{K}_{\text {ext }}\left(\theta_{\text {inc }}, D\right)$ is described in Eq. (12), where

$$
\begin{gathered}
Q_{\mathrm{pol}}\left(\theta_{\mathrm{inc}}, D\right)=\frac{\sigma_{\mathrm{pol}}\left(\theta_{\mathrm{inc}}, D\right)}{\sigma_{\mathrm{ext}}\left(\theta_{\mathrm{inc}}, D\right)}, \\
Q_{\mathrm{cpol}}\left(\theta_{\mathrm{inc}}, D\right)=\frac{\sigma_{\mathrm{cpol}}\left(\theta_{\mathrm{inc}}, D\right)}{\sigma_{\mathrm{ext}}\left(\theta_{\mathrm{inc}}, D\right)} .
\end{gathered}
$$

\section{APPENDIX B}

\section{A Conversion from the Laboratory to Image Coordinate}

The rotation of coordinate systems follows Eq. (1) but replacing the laboratory coordinate system with the horizontal coordinate system (the $x_{w}, y_{w}$, and $z_{w}$ axes are pointing in the south, east, and zenith directions on the local Earth's surface), and the particle coordinate system with the camera coordinate system (the $x_{c}, y_{c}$, and $z_{c}$ axes are pointing in the right, downward, and forward directions in reference to the pointing direction of the camera). Then the camera coordinate system is projected on a two-dimensional image coordinate $(u, v)$, with its origin at the left-top pixel. In the image coordinate, the pixel aspect ratio is assumed to be unity. The vector components on the image coordinate are

$$
\begin{gathered}
u=\frac{N_{u}}{2}\left[\frac{\mathbf{C}\left(\theta_{c}\right)}{\mathbf{C}\left(\theta_{c, \text { max }}\right)} \frac{\sqrt{N_{u}^{2}+N_{v}^{2}}}{\sqrt{x_{c}^{2}+y_{c}^{2}}} \frac{x_{c}}{N_{u}}+1\right], \\
v=\frac{N_{v}}{2}\left[\frac{\mathbf{C}\left(\theta_{c}\right)}{\mathbf{C}\left(\theta_{c, \text { max }}\right)} \frac{\sqrt{N_{u}^{2}+N_{v}^{2}}}{\sqrt{x_{c}^{2}+y_{c}^{2}}} \frac{y_{c}}{N_{v}}+1\right],
\end{gathered}
$$

where $N_{u}$ and $N_{v}$ are the numbers of pixels along the $u$ and $v$ directions. The equisolid-angle projection is determined based on the camera's half angle of view $\theta_{c}$ in the form

$$
\mathbf{C}\left(\theta_{c}\right)=2 \sin \theta_{c} / 2,
$$

where

$$
\sin \theta_{c}=\sqrt{x_{c}^{2}+y_{c}^{2}} .
$$

To conduct numerical computation based on these mapping formulas, we modify a function in the Monte Carlo Atmospheric Radiative Transfer Simulator (MCARaTS; Iwabuchi 2006).

\section{REFERENCES}

Baum, B. A., P. Yang, A. J. Heymsfield, S. Platnick, M. D. King, and S. T. Bedka, 2005: Bulk scattering models for the remote sensing of ice clouds. Part II: Narrowband models. J. Appl. Meteor., 44, 1896-1911, https://doi.org/10.1175/JAM2309.1.

Bi, L., and P. Yang, 2014: Accurate simulation of the optical properties of atmospheric ice crystals with the invariant imbedding T-matrix method. J. Quant. Spectrosc. Radiat. Transfer, 138, 17-35, https://doi.org/10.1016/j.jqsrt.2014.01.013.

$\longrightarrow$, and,- 2017 : Improved ice particle optical property simulations in the ultraviolet to far-infrared regime. J. Quant. Spectrosc. Radiat. Transfer, 189, 228-237, https://doi.org/ 10.1016/j.jqsrt.2016.12.007.

,-- , and G. W. Kattawar, 2010: Edge-effect contribution to the extinction of light by dielectric disk and cylindrical particles. Appl. Opt., 49, 4641-4646, https://doi.org/10.1364/ AO.49.004641.

,,,--- Y. Hu, and B. A. Baum, 2011: Scattering and absorption of light by ice particles: Solution by a new physical-geometric optics hybrid method. J. Quant. Spectrosc. Radiat. Transfer, 112, 1492-1508, https://doi.org/ 10.1016/j.jqsrt.2011.02.015.

,,--- , and M. I. Mishchenko, 2013: Efficient implementation of the invariant imbedding T-matrix method and the separation of variables method applied to large nonspherical inhomogeneous particles. J. Quant. Spectrosc. Radiat. Transfer, 116, 169-183, https://doi.org/10.1016/j.jqsrt.2012.11.014.

Borovoi, A., A. Konoshonkin, N. Kustova, and H. Okamoto, 2012: Backscattering Mueller matrix for quasi-horizontally oriented ice plates of cirrus clouds: Application to CALIPSO signals. Opt. Express, 20, 28 222-28 233, https://doi.org/ 10.1364/OE.20.028222.

,$- \ldots$, and -2014 : The physical-optics approximation and its application to light backscattering by hexagonal ice crystals. J. Quant. Spectrosc. Radiat. Transfer, 146, 181-189, https:// doi.org/10.1016/j.jqsrt.2014.04.030.

Bréon, F.-M., and B. Dubrulle, 2004: Horizontally oriented plates in clouds. J. Atmos. Sci., 61, 2888-2898, https://doi.org/10.1175/ JAS-3309.1.

Cai, Q., and K. N. Liou, 1982: Polarized light scattering by hexagonal ice crystals: Theory. Appl. Opt., 21, 3569-3580, https:// doi.org/10.1364/AO.21.003569.

Chepfer, H., G. Brogniez, P. Goloub, M. B. Francois, and P. H. Flamant, 1999: Observations of horizontally oriented ice crystals in cirrus clouds with POLDER-1/ADEOS-1. J. Quant. Spectrosc. Radiat. Transfer, 63, 521-543, https://doi.org/10.1016/ S0022-4073(99)00036-9.

Ding, J., L. Bi, P. Yang, G. L. Kattawar, F. Weng, Q. Liu, and T. Greenwald, 2017: Single-scattering properties of ice 
particles in the microwave regime: Temperature effect on the ice refractive index with implications in remote sensing. J. Quant. Spectrosc. Radiat. Transfer, 190, 26-37, https:// doi.org/10.1016/j.jqsrt.2016.11.026.

Forster, L., M. Seefeldner, M. Wiegner, and B. Mayer, 2017: Ice crystal characterization in cirrus clouds: A sun-tracking camera system and automated detection algorithm for halo displays. Atmos. Meas. Tech., 10, 2499-2516, https://doi.org/ 10.5194/amt-10-2499-2017.

Fröhlich, C., and G. E. Shaw, 1980: New determination of Rayleigh scattering in the terrestrial atmosphere. Appl. Opt., 19, 17731775, https://doi.org/10.1364/AO.19.001773.

Greenler, R. G., 1980: Rainbows, Halos, and Glories. Cambridge University Press, $240 \mathrm{pp}$.

— , and A. J. Mallmann, 1972: Circumscribed halos. Science, 176, 128-131, https://doi.org/10.1126/science.176.4031.128.

Hashino, T., M. Chiruta, D. Polzin, A. Kubicek, and P. K. Wang, 2014: Numerical simulation of the flow fields around falling ice crystals with inclined orientation and the hydrodynamic torque. Atmos. Res., 150, 79-96, https://doi.org/10.1016/ j.atmosres.2014.07.003.

—, K.-Y. Cheng, C.-C. Chueh, and P. K. Wang, 2016: Numerical study of motion and stability of falling columnar crystals. J. Atmos. Sci., 73, 1923-1942, https://doi.org/10.1175/JAS-D-15-0219.1.

Hu, Y., D. Winker, P. Yang, B. A. Baum, L. Poole, and L. Vann, 2001: Identification of cloud phase from PICASSO-CENA lidar depolarization: A multiple scattering sensitivity study. J. Quant. Spectrosc. Radiat. Transfer, 70, 569-579, https:// doi.org/10.1016/S0022-4073(01)00030-9.

—_, and Coauthors, 2009: CALIPSO/CALIOP cloud phase discrimination algorithm. J. Atmos. Oceanic Technol., 26, 2293 2309, https://doi.org/10.1175/2009JTECHA1280.1.

Illingworth, A. J., and Coauthors, 2015: The EarthCARE satellite: The next step forward in global measurements of clouds, aerosols, precipitation, and radiation. Bull. Amer. Meteor. Soc., 96, 1311-1332, https://doi.org/10.1175/BAMS-D-12-00227.1.

Iwabuchi, H., 2006: Efficient Monte Carlo methods for radiative transfer modeling. J. Atmos. Sci., 63, 2324-2339, https:// doi.org/10.1175/JAS3755.1.

—_ P. Yang, K. Liou, and P. Minnis, 2012: Physical and optical properties of persistent contrails: Climatology and interpretation. J. Geophys. Res., 117, D06215, https://doi.org/10.1029/ 2011JD017020.

Johnson, B. R., 1988: Invariant imbedding T matrix approach to electromagnetic scattering. Appl. Opt., 27, 4861-4873, https:// doi.org/10.1364/AO.27.004861.

Kuo, K.-S., and Coauthors, 2016: The microwave radiative properties of falling snow derived from nonspherical ice particle models. Part I: An extensive database of simulated pristine crystals and aggregate particles, and their scattering properties. J. Appl. Meteor. Climatol., 55, 691-708, https://doi.org/ 10.1175/JAMC-D-15-0130.1.

Lawson, R. P., B. Baker, B. Pilson, and Q. Mo, 2006: In situ observations of the microphysical properties of wave, cirrus, and anvil clouds. Part II: Cirrus clouds. J. Atmos. Sci., 63, 31863203, https://doi.org/10.1175/JAS3803.1.

Liou, K. N., 1986: Influence of cirrus clouds on weather and climate processes: A global perspective. Mon. Wea. Rev., 114, 1167-1199, https://doi.org/10.1175/1520-0493(1986)114<1167: IOCCOW $>2.0 . \mathrm{CO} ; 2$.

— mentals and Applications. 1st ed. Cambridge University Press, 443 pp.
Liu, G., 2008: A database of microwave single-scattering properties for nonspherical ice particles. Bull. Amer. Meteor. Soc., 89, 1563-1570, https://doi.org/10.1175/2008BAMS2486.1.

Lohmann, U., and E. Roeckner, 1995: Influence of cirrus cloud radiative forcing on climate and climate sensitivity in a general circulation model. J. Geophys. Res., 100, 16305-16323, https:// doi.org/10.1029/95JD01383.

Lynch, D. K., and W. Livingston, 2001: Color and Light in Nature. 2nd ed. Cambridge University Press, 277 pp.

Marshak, A., T. Vaìnai, and A. Kostinski, 2017: Terrestrial glint seen from deep space: Oriented ice crystals detected from the Lagrangian point. Geophys. Res. Lett., 44, 5197-5202, https:// doi.org/10.1002/2017GL073248.

Mishchenko, M. I., 1991: Extinction and polarization of transmitted light by partially aligned nonspherical grains. Astrophys. J., 367, 561-574, https://doi.org/10.1086/169652.

_ , and L. D. Travis, 1994: T-matrix computations of light scattering by large spheroidal particles. Opt. Commun., 109, 1621, https://doi.org/10.1016/0030-4018(94)90731-5.

Noel, V., and K. Sassen, 2005: Study of planar ice crystal orientations in ice clouds from scanning polarization lidar observations. J. Appl. Meteor., 44, 653-664, https://doi.org/10.1175/ JAM2223.1.

—, H. Chepfer, G. Ledanois, and P. H. Flamant, 2001: Computation of a single-scattering matrix for nonspherical particles randomly or horizontally oriented in space. Appl. Opt., 40, 4365-4375, https://doi.org/10.1364/AO.40.004365.

Okamoto, H., K. Sato, and Y. Hagihira, 2010: Global analysis of ice microphysics from CloudSat and CALIPSO: Incorporation of specular reflection in lidar signals. J. Geophys. Res., 115, D22209, https://doi.org/10.1029/2009JD013383.

Pattloch, F., and E. Tränkle, 1984: Monte Carlo simulation and analysis of halo phenomena. J. Opt. Soc. Amer., 1A, 520-526, https://doi.org/10.1364/JOSAA.1.000520.

Platnick, S., M. D. King, S. A. Ackerman, W. P. Menzel, B. A. Baum, J. C. Rieìdi, and R. A. Frey, 2003: The MODIS cloud products: Algorithms and examples from Terra. IEEE Trans. Geosci. Remote Sens., 41, 459-473, https://doi.org/10.1109/ TGRS.2002.808301.

Purcell, E. M., and C. R. Pennypacker, 1973: Scattering and absorption of light by nonspherical dielectric grains. Astrophys. J., 186, 705-714, https://doi.org/10.1086/152538.

Saito, M., H. Iwabuchi, P. Yang, G. Tang, M. D. King, and M. Sekiguchi, 2017: Ice particle morphology and microphysical properties of cirrus clouds inferred from combined CALIOP-IIR measurements. J. Geophys. Res. Atmos., 122, 4440-4462, https://doi.org/10.1002/2016JD026080.

Sassen, K., 1991: The polarization lidar technique for cloud research: A review and current assessment. Bull. Amer. Meteor. Soc., 72, 1848-1866, https://doi.org/10.1175/1520-0477(1991) 072<1848:TPLTFC $>2.0$.CO;2.

— matology from the Facility for Atmospheric Remote Sensing. Part III: Radiative properties. J. Atmos. Sci., 58, 2113-2127, https://doi.org/10.1175/1520-0469(2001)058<2113:AMCCCF> 2.0.CO;2.

— J. Zhu, and S. Benson, 2003: A midlatitude cirrus cloud climatology from the Facility for Atmospheric Remote Sensing: IV. Optical displays. Appl. Opt., 42, 332-341, https://doi.org/ 10.1364/AO.42.000332.

_ clouds from CloudSat/Cloud-Aerosol Lidar and Infrared Pathfinder Satellite Observations (CALIPSO) measurements. 
J. Geophys. Res., 113, D00A12, https://doi.org/10.1029/ 2008JD009972.

Sun, B., P. Yang, G. W. Kattawar, and X. Zhang, 2017: Physicalgeometric optics method for large size faceted particles. Opt. Express, 25, 24 044-24 060, https://doi.org/10.1364/OE.25.024044.

Takano, Y., and K. N. Liou, 1989: Solar radiative transfer in cirrus clouds. Part II: Theory and computation of multiple scattering in an anisotropic medium. J. Atmos. Sci., 46, 20-36, https://doi.org/ 10.1175/1520-0469(1989)046<0020:SRTICC $>2.0$. CO;2.

$\longrightarrow$, and $—$, 1993: Transfer of polarized infrared radiation in optically anisotropic media: Application to horizontally oriented crystals. J. Opt. Soc. Amer., 10A, 1243-1256, https:// doi.org/10.1364/JOSAA.10.001243.

Voss, K. J., and Y. Liu, 1997: Polarized radiance distribution measurements of skylight. I. System description and characterization. Appl. Opt., 36, 6083-6094, https://doi.org/10.1364/ AO.36.006083.

Waliser, D. E., and Coauthors, 2009: Cloud ice: A climate model challenge with signs and expectations of progress. J. Geophys. Res., 114, D00A21, https://doi.org/10.1029/2008JD010015.

Warren, S. G., and R. E. Brandt, 2008: Optical constants of ice from the ultraviolet to the microwave: A revised compilation. J. Geophys. Res., 113, D14220, https://doi.org/10.1029/ 2007JD009744.

Waterman, P., 1965: Matrix formulation of electromagnetic scattering. Proc. IEEE, 53, 805-812, https://doi.org/10.1109/ PROC.1965.4058.

Winker, D. M., M. A. Vaughan, A. Omar, Y. Hu, K. A. Powell, Z. Liu, W. H. Hunt, and S. A. Young, 2009: Overview of the CALIPSO mission and CALIOP data processing algorithms. J. Atmos. Oceanic Technol., 26, 2310-2323, https://doi.org/ 10.1175/2009JTECHA1281.1.

Yang, P., and K. N. Liou, 1996: Geometric-optics-integral-equation method for light scattering by nonspherical ice crystals. Appl. Opt., 35, 6568-6584, https://doi.org/10.1364/AO.35.006568.

- and - 1997: Light scattering by hexagonal ice crystals: Solutions by a ray-by-ray integration algorithm. J. Opt. Soc. Amer., 14, 2278-2289, https://doi.org/10.1364/JOSAA.14.002278.

-, Y. X. Hu, D. M. Winker, J. Zhao, C. A. Hosteller, B. A. Baum, M. I. Mishchenko, and J. Reichardt, 2003: Enhanced lidar backscattering by horizontally oriented ice plates. J. Quant. Spectrosc. Radiat. Transfer, 79-80, 11391157, https://doi.org/10.1016/S0022-4073(02)00346-1.

—, L. Bi, B. A. Baum, K. N. Liou, G. L. Kattawar, M. I. Mishchenko, and B. Cole, 2013: Spectrally consistent scattering, absorption, and polarization properties of atmospheric ice crystals at wavelengths from 0.2 to $100 \mu \mathrm{m}$. J. Atmos. Sci., 70, 330-347, https://doi.org/10.1175/JAS-D-12-039.1.

- K.-N. Liou, L. Bi, C. Liu, B. Yi, and B. A. Baum, 2015: On the radiative properties of ice clouds: Light scattering, remote sensing, and radiation parameterization. Adv. Atmos. Sci., 32, 32-63, https://doi.org/10.1007/s00376-014-0011-z.

_- S. Hioki, M. Saito, C.-P. Kuo, B. A. Baum, and K.-N. Liou, 2018: A review of ice cloud optical property models for passive satellite remote sensing. Atmosphere, 9, 499, https://doi.org/ 10.3390/atmos9120499.

_ , J. Ding, R. L. Panetta, K.-N. Liou, G. W. Kattawar, and M. I. Mishchenko, 2019: On the convergence of numerical computations for both exact and approximate solutions for electromagnetic scattering by nonspherical dielectric particles. Prog. Electromagn. Res., 164, 27-61, https://doi.org/ 10.2528/PIER18112810.

Yee, S. K., 1966: Numerical solution of initial boundary value problems involving Maxwell's equation in isotropic media. IEEE Trans. Antennas Propag., 14, 302-307, https://doi.org/ 10.1109/TAP.1966.1138693.

Yoshida, R., H. Okamoto, Y. Hagihara, and H. Ishimoto, 2010: Global analysis of cloud phase and ice crystal orientation from Cloud-Aerosol Lidar and Infrared Pathfinder Satellite Observation (CALIPSO) data using attenuated backscattering and depolarization ratio. J. Geophys. Res., 115, D00H32, https://doi.org/10.1029/2009JD012334.

Yurkin, M. A., V. P. Maltsev, and A. G. Hoekstra, 2007: The discrete dipole approximation for simulation of light scattering by particles much larger than the wavelength. J. Quant. Spectrosc. Radiat. Trans., 106, 546-557, https:// doi.org/10.1016/j.jqsrt.2007.01.033.

Zhou, C., P. Yang, A. E. Dessler, Y. Hu, and B. A. Baum, 2012: Study of horizontally oriented ice crystals with CALIPSO observations and comparison with Monte Carlo radiative transfer simulations. J. Appl. Meteor. Climatol., 51, 14261439, https://doi.org/10.1175/JAMC-D-11-0265.1. 